\title{
CONVERGENCE ANALYSIS FOR AN EXPONENTIALLY FITTED FINITE VOLUME METHOD
}

\author{
REINER VANSELOW ${ }^{1}$
}

\begin{abstract}
The paper is devoted to the convergence analysis of a well-known cell-centered Finite Volume Method (FVM) for a convection-diffusion problem in $\mathbb{R}^{2}$. This FVM is based on Voronoi boxes and exponential fitting. To prove the convergence of the FVM, we use a new nonconforming PetrovGalerkin Finite Element Method (FEM) for which the system of linear equations coincides completely with that of the FVM. Thus, by proving convergence properties of the FEM we obtain similar ones for the FVM. For the error estimation of the FEM well-known statements have to be modified.
\end{abstract}

Mathematics Subject Classification. 65N12, 65N99, 65N30.

Received: February 16, 2000. Revised: June 5, 2000 and July 17, 2000.

\section{Introduction}

In this paper we analyze the convergence of a cell-centered FVM applied to the convection-diffusion boundary value problem:

$$
-\operatorname{div}[\varepsilon \operatorname{grad} u+b u]=f \quad \text { in } \Omega \subset \mathbb{R}^{2}, \quad u=0 \quad \text { on } \Gamma=\partial \Omega .
$$

The coefficient $\varepsilon$ is a positive parameter, $b$ is a given constant vector, $f \in L_{2}(\Omega)$ is a given function and $\Omega$ is an open and bounded domain with a smooth boundary. In order to simplify the presentation we restrict ourselves to a convex domain $\Omega$ and to homogeneous Dirichlet boundary conditions.

For an equation in the conservative form (1.1), FVMs are often used, because these methods obviously conserve a significant property of the exact solution (see Rem. 3.2).

The FVM considered in our paper uses Voronoi boxes and is based on a finite difference approximation, which is exact for functions that solve the ordinary differential equation $\varepsilon u^{\prime \prime}+\left(e^{T} b\right) u^{\prime}=0$ along a straight line ( $e$ denotes the unit vector in the direction of this straight line). This FVM is well-known and is successfully used in different applications, e.g. for the numerical solution of the semiconductor equations (cf. e.g. [2]).

Convergence results for FVMs applied to the convection-diffusion equation (1.1) are given in discrete norms e.g. in [10-12]. In contrast to those papers, we follow another approach and prove the convergence of a FVM by analyzing a corresponding FEM as e.g. in [23] for the Poisson equation. This gives convergence results in stronger norms (see Rem. 4.2).

Keywords and phrases. Convection-diffusion problem, cell-centered finite volume method, Voronoi boxes, exponential fitting, convergence analysis, nonconforming finite element method.

1 Dresden University of Technology, Department of Mathematics, 01062 Dresden, Germany.

e-mail: vanselow@math.tu-dresden.de 
Furthermore, it is well-known that a FVM can be interpreted as a FEM, if suitable ansatz and test functions are used. We consider a new FEM, which generalizes that given in [23] for the Poisson equation. The advantage of this FEM is that it provides an error estimate, if we follow the methodology given e.g. in [6], which consists of applying the second Strang lemma and usual techniques for the estimation of the approximation and consistency error terms. Differences with respect to other FEMs for convection-diffusion equations and their analysis are discussed later.

However, modifications of some well-known statements are necessary to make the approach workable. One of these modifications concerns the second Strang lemma because we use a Petrov-Galerkin FEM. Furthermore, because we use exponentially fitted ansatz functions, we substitute the vector space $P_{1}(\Delta)$ of polynomials of first degree and the seminorm $|\cdot|_{2, \Delta}$ of the Sobolev space $H^{2}(\Delta)$ by other ones, where $\Delta$ denotes an arbitrary finite element or finite volume (see Sects. 6.3 and 6.4). This modification is fundamental for the estimation of the error terms.

To obtain constants independent of $\varepsilon$ in the estimates, for $h$ in general we assume an upper bound which depends on $\varepsilon$ (see (6.2)). This is a restrictive assumption for singularly perturbed problems, where $\varepsilon$ is small. However, we do not need any information about the location or the type of boundary layers in the singular perturbed case. This information is necessary in the standard analysis of singular perturbed problems.

An overview of discretization methods for the problem (1.1) and the corresponding analysis is given in [14,16]. Here, we want to discuss only some references in detail which are closely related to our paper.

In $[1,17]$ FEMs are considered which lead to systems of linear equations whose coefficient matrices are different with respect to the one discussed in our paper. However, our approach coincides in some details with those ones.

In [1] a Galerkin FEM is analyzed which uses triangles and conforming linear finite elements. The estimates are given in the $H^{1}$-seminorm, which implies a stronger convergence result. However, an important conservation property of FVMs is in general not satisfied by the discretization obtained by Angermann [1] (see Rem. 3.3). Thus, the results deduced for that FEM cannot be in general extended to FVMs.

Another approach is given by Sacco and Stynes [17]. They point out the well-known fact that good results are obtained when using a FEM whose trial functions lie locally in the null space of the differential operator. These functions are the so-called L-splines ( $c f$. [20]). To extend this approach to the two-dimensional case with a given triangulation, in [17] a new nonconforming Petrov-Galerkin FEM is considered. The test functions are polynomials. The ansatz functions are L-splines in the direction of the vector $b$ and linear functions in the direction which is orthogonal to $b$. A corresponding convergence analysis for that FEM is given in [18].

In the following references FEMs are investigated which lead to systems of linear equations, where the coefficient matrices are the same as in our paper.

In [12] a nonconforming Petrov-Galerkin FEM is considered, too. However, as mentioned above, a weaker norm is used, which only results in an error estimate for the difference between the FEM solution $u_{h}$ and an interpolant of the exact solution $u$.

In $[8,25]$ new FEMs are presented and analyzed, which use triangles and conforming linear finite elements. Unlike the present paper, the estimates are given in other norms and other techniques are used to obtain those estimates (see Rem. 5.1).

Bank et al. [3] extend the results of Xu and Zikatanov [25] to a FVM, which is the same as that in our paper.

Our paper is organized as follows. In Section 2 a new nonconforming FEM is introduced. The FVM for which the convergence analysis is presented and its relations to the new FEM are described in Section 3. Section 4 gives the application of the second Strang lemma. The convergence theorem, which is the main result of this paper, is presented in Section 5. In Section 6 assumptions and useful tools are given, which are needed in Section 7 to estimate the different error terms.

More details about the partitions of the domain $\Omega$ (see Sect. 2.1), the assumption (A) (see Sect. 2.1) and the affine mapping defined in Section 6.2 can be found in [23]. 


\section{A nonconforming Petrov-Galerkin FEM}

\subsection{Box and dual box partition}

In the following, let $M:=\{P\}$ be a finite set of given points with $P \in \mathrm{Cl}(\Omega)$. Further, we use the notations $M_{i}:=M \cap \Omega$ and $M_{b}:=M \cap \Gamma$, where $m:=\operatorname{card}\left(M_{i}\right)>0$ and card $\left(M_{b}\right)>2$ have to be satisfied.

Now, for $P \in M$ the Voronoi box $b_{P}$ is defined by

$$
b_{P}:=\{Z \in \mathrm{Cl}(\Omega):|Z-P| \leq|Z-Q| \forall Q \in M\},
$$

and the set $B_{h}:=B_{h}(M):=\left\{b_{P}: P \in M\right\}$ is called box partition of $\Omega$.

If for different points $P, Q \in M$ the intersection $b_{P} \cap b_{Q}$ is non-empty, the endpoints of $b_{P} \cap b_{Q}$ are denoted by $E_{1}=E_{1}(P, Q)$ and $E_{2}=E_{2}(P, Q)$, i.e. $\overline{E_{1} E_{2}}=b_{P} \cap b_{Q}$.

For $P \in M_{i}$ we use the notations

$$
\begin{aligned}
& N(P):=\left\{Q \in M: Q \neq P, b_{P} \cap b_{Q} \neq \emptyset\right\}, \\
& \beta_{P Q}:=|P-Q| \text { and } \gamma_{P Q}:=\left|E_{1}-E_{2}\right| \text { for all } Q \in N(P), \\
& N N(P):=\left\{Q \in N(P): \gamma_{P Q}>0\right\} \text { and } N N_{i}(P):=N N(P) \cap \Omega .
\end{aligned}
$$

Further, we need another partition of the domain $\Omega$. Therefore, for $P \in M_{i}$ and $Q \in N N(P)$ the dual Voronoi box $d b_{P Q}$ is defined by

$$
d b_{P Q}:=\triangle E_{1} P E_{2} \cup \triangle E_{1} Q E_{2},
$$

and the set $d B_{h}:=d B_{h}(M):=\left\{d b_{P Q}: P \in M_{i}\right.$ and $\left.Q \in N N(P)\right\}$ is called dual box partition of $\Omega$.

Henceforth, we assume that the following property is satisfied:

$$
\oint_{\partial b_{P}} \mathrm{~d} \Gamma=\sum_{Q \in N N(P)} \int_{b_{P} \cap b_{Q}} \mathrm{~d} \Gamma \quad \forall P \in M_{i} .
$$

\subsection{Description of the FEM}

A weak formulation of the boundary value problem (1.1) reads as follows:

Find $u \in V:=H_{0}^{1}(\Omega)$ such that

$$
a(u, v):=\iint_{\Omega}(\varepsilon \operatorname{grad} u+b u)^{T} \operatorname{grad} v \mathrm{~d} \Omega=\iint_{\Omega} f v \mathrm{~d} \Omega=: d(v) \quad \forall v \in V .
$$

For the new Petrov-Galerkin FEM we define finite-dimensional vector spaces $V_{h}^{1}$ and $V_{h}^{2}$ by

$$
V_{h}^{1}:=\left\{v \in V_{h}:\left.v\right|_{\operatorname{Int}\left(d b_{P Q}\right)} \in L K_{\varepsilon}(P, Q)\right\}
$$

and

$$
V_{h}^{2}:=\left\{v \in V_{h}:\left.v\right|_{\operatorname{Int}\left(d b_{P Q}\right)} \in \widetilde{P}(P, Q)\right\}
$$

with $V_{h}:=\left\{v \in L_{2}(\Omega): v\right.$ is continuous at $P \in M$ and $\left.v(P)=0 \quad \forall P \in M_{b}\right\}$.

Thereby, $L K_{\varepsilon}(P, Q)$ and $\widetilde{P}(P, Q)$ with $P=\left(x_{P}, y_{P}\right)^{T}, Q=\left(x_{Q}, y_{Q}\right)^{T}, P \neq Q$,

$$
F_{1, \varepsilon}(x, y):=\exp \left(\frac{\left[e_{P Q}\right]^{T} b}{\varepsilon \beta_{P Q}} F_{2}(x, y)\right), \quad F_{2}(x, y):=\left[\left(x_{P}-x_{Q}\right)\left(x-x_{P}\right)+\left(y_{P}-y_{Q}\right)\left(y-y_{P}\right)\right]
$$


and the vector

$$
e_{P Q}:=\frac{Q-P}{|Q-P|}=\frac{1}{\beta_{P Q}}\left(x_{Q}-x_{P}, y_{Q}-y_{P}\right)^{T},
$$

denote the vector spaces

$$
L K_{\varepsilon}(P, Q):= \begin{cases}\operatorname{span}\left\{1, F_{1, \varepsilon}(x, y)\right\}, & \text { if }\left[e_{P Q}\right]^{T} b \neq 0 \\ \operatorname{span}\left\{1, F_{2}(x, y)\right\}, & \text { if }\left[e_{P Q}\right]^{T} b=0\end{cases}
$$

and

$$
\widetilde{P}(P, Q):=\operatorname{span}\left\{1, F_{2}(x, y)\right\}
$$

As degrees of freedom we choose the function values at the points $P \in M_{i}$.

Obviously, $\operatorname{dim}\left(V_{h}^{i}\right)=m$ and $V_{h}^{i} \not \subset V, i=1,2$.

Remark 2.1. In Int $\left(d b_{P Q}\right)$ the following characterization is true:

$$
v_{h} \in V_{h}^{1}:\left\{\begin{array}{l}
\text { the function which in the direction of } P-Q \text { is the solution of the } \\
\text { ordinary BVP } \varepsilon u^{\prime \prime}+\beta_{P Q}\left(\left[e_{P Q}\right]^{T} b\right) u^{\prime}=0, u(0)=u_{P}, u(1)=u_{Q} \\
\text { (L-spline) and which is constant on lines orthogonal to } P-Q
\end{array}\right.
$$

Let us now consider the nonconforming Petrov-Galerkin FEM:

Find $u_{h}=u_{h}(M) \in V_{h}^{1}$ such that

$$
a_{h}\left(u_{h}, v_{h}\right)=\frac{1}{2} \sum_{R \in M_{i}} v_{h}(R) D(R)=: d_{h}\left(v_{h}\right) \quad \forall v_{h} \in V_{h}^{2}
$$

with

$$
a_{h}\left(u_{h}, v_{h}\right):=\sum_{d b_{P Q} \in d B_{h}} \iint_{d b_{P Q}}\left(\varepsilon \operatorname{grad} u_{h}+b u_{h}\right)^{T} \operatorname{grad} v_{h} \mathrm{~d} \Omega
$$

which is defined on $\left[V \oplus V_{h}^{1}\right] \times V_{h}^{2}$, and

$$
D(P):=\iint_{b_{P}} f \mathrm{~d} \Omega
$$

The nodal basis functions $\Phi$ and $\Psi$ of $V_{h}^{1}$ and $V_{h}^{2}$ are respectively given by

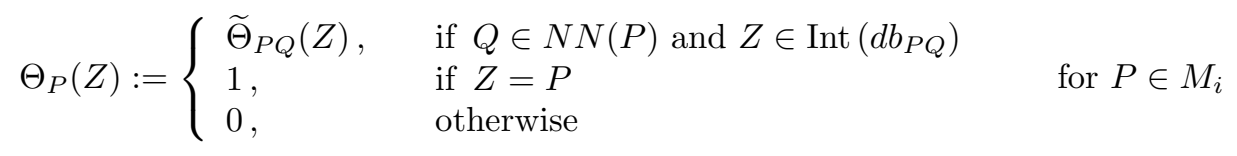


with $\Theta \in\{\Phi, \Psi\}, Z=(x, y)^{T}$,

$$
\widetilde{\Phi}_{P Q}(Z):= \begin{cases}\frac{1}{\exp \left(\frac{\beta_{P Q}}{\varepsilon}\left[e_{P Q}\right]^{T} b\right)-1}\left[-1+\exp \left(\frac{\beta_{P Q}}{\varepsilon}\left[e_{P Q}\right]^{T} b\right) F_{1, \varepsilon}(x, y)\right], & \text { if }\left[e_{P Q}\right]^{T} b \neq 0 \\ 1+\frac{1}{\left[\beta_{P Q}\right]^{2}} F_{2}(x, y), & \text { if }\left[e_{P Q}\right]^{T} b=0\end{cases}
$$

and

$$
\widetilde{\Psi}_{P Q}(Z):=1+\frac{1}{\left[\beta_{P Q}\right]^{2}} F_{2}(x, y)
$$

Using the nodal basis a system of linear equations arises, which has the form

$$
L^{E} u^{E}=b^{E} .
$$

The stiffness matrix $L^{E}$, the vector $u^{E}=u^{E}(M) \in \mathbb{R}^{m}$ and the right-hand side $b^{E}$ are given by

$$
L_{P Q}^{E}=a_{h}\left(\Phi_{Q}, \Psi_{P}\right), \quad u_{P}^{E}=u_{h}(P) \quad \text { and } \quad b_{P}^{E}=d_{h}\left(\Psi_{P}\right) \quad \text { for } P, Q \in M_{i} .
$$

\section{THE FVM}

\subsection{Description of the FVM}

If we integrate both sides of (1.1) over the Voronoi box $b_{p} \in B_{h}$, apply Green's formula and use a relation like (A), we obtain the equations

$$
-\sum_{Q \in N N(P)} \int_{b_{P} \cap b_{Q}}\left[e_{P Q}\right]^{T}(\varepsilon \operatorname{grad} u+b u) \mathrm{d} \Gamma=D(P) \quad \forall P \in M_{i},
$$

where $e_{P Q}$ and $D(P)$ are defined by (2.4) and (2.9), respectively.

The integrand $\left[e_{P Q}\right]^{T}(\varepsilon \operatorname{grad} u+b u)$ is then substituted on each straight line $b_{P} \cap b_{Q}$ by the constant finite difference approximation

$$
\left[e_{P Q}\right]^{T}(\varepsilon \operatorname{grad} u+b u) \approx \frac{1}{\beta_{P Q}}\left\{B\left(-\frac{\beta_{P Q}}{\varepsilon}\left[e_{P Q}\right]^{T} b\right) u(Q)-B\left(\frac{\beta_{P Q}}{\varepsilon}\left[e_{P Q}\right]^{T} b\right) u(P)\right\}
$$

(cf. e.g. [21]), where the Bernoulli function $B$ is defined by

$$
B(z):= \begin{cases}\frac{z}{\exp (z)-1}, & \text { if } z \neq 0 \\ 1, & \text { if } z=0 .\end{cases}
$$

Remark 3.1. The approximation (3.2) is exact for functions $u \in L K_{\varepsilon}(P, Q)$.

(3.1) and (3.2) give rise to the following well-known FVM (cf. e.g. [2]):

$$
\text { Find } u^{V}=u^{V}(M) \in \mathbb{R}^{m} \text { such that } L^{V} u^{V}=b^{V},
$$


where the matrix $L^{V}$ and the right-hand side $b^{V}$ are given for $P, Q \in M_{i}$ by

$$
L_{P Q}^{V}=\left\{\begin{array}{ll}
-\varepsilon \frac{\gamma_{P Q}}{\beta_{P Q}} B\left(-\frac{\beta_{P Q}}{\varepsilon}\left[e_{P Q}\right]^{T} b\right), & \text { if } Q \in N N_{i}(P) \\
\sum_{R \in N N(P)} \varepsilon \frac{\gamma_{P R}}{\beta_{P R}} B\left(\frac{\beta_{P R}}{\varepsilon}\left[e_{P R}\right]^{T} b\right), & \text { if } Q=P \\
0, & \text { otherwise }
\end{array} \quad \text { and } b_{P}^{V}=D(P) .\right.
$$

Remark 3.2. For convection-diffusion problems in conservative form (1.1) the following property of the exact solution is significant: We consider an arbitrary polygonal partition $\mathcal{T}_{h}=\{t\}$ of the domain $\Omega$, i.e. $t$ is bounded by a polygon for all $t \in \mathcal{T}_{h}$ and it holds $\mathrm{Cl}(\Omega)=\bigcup_{t \in \mathcal{T}_{h}} \mathrm{Cl}(t)$ as well as $t_{1} \cap t_{2}=\emptyset$ for all $t_{1}, t_{2} \in \mathcal{T}_{h}$ with $t_{1} \neq t_{2}$. Further, we define the flux $F$ by $F(u):=\varepsilon \operatorname{grad} u+b u$ and denote the outer normal direction of $t \in \mathcal{T}_{h}$ by $n(t)$. Then for two arbitrary elements $t_{1}, t_{2} \in \mathcal{T}_{h}$ with $t_{1} \neq t_{2}$ and $\Gamma\left(t_{1}, t_{2}\right):=\partial t_{1} \cap \partial t_{2} \neq \emptyset$ the outflow of $t_{1}$ through $\Gamma\left(t_{1}, t_{2}\right)$, which is defined by $\int_{\Gamma\left(t_{1}, t_{2}\right)}\left[n\left(t_{1}\right)\right]^{T} F(u) \mathrm{d} \Gamma$, is equal to the inflow of $t_{2}$ through $\Gamma\left(t_{1}, t_{2}\right)$, which is defined by $-\int_{\Gamma\left(t_{1}, t_{2}\right)}\left[n\left(t_{2}\right)\right]^{T} F(u) \mathrm{d} \Gamma$.

Obviously, the FVM (3.4) conserves that property for the box partition.

\subsection{Relations between the FVM and the nonconforming FEM}

Theorem 3.1. The matrices $L^{E}$ and $L^{V}$ of the systems of linear equations (2.12) and (3.4) are related by $L^{E}=\frac{1}{2} L^{V}$.

Proof. We consider points $P, Q \in M_{i}$ and have to distinguish three cases: $Q \in N N_{i}(P), Q \notin N N_{i}(P)$ with $Q \neq P$ and $P=Q$. Straightforward calculations for each of these cases prove the statement.

For instance, in the case $Q \in N N_{i}(P)$ we obtain

$$
L_{P Q}^{E}=a_{h}\left(\Phi_{Q}, \Psi_{P}\right)=\iint_{d b_{P Q}}\left(\varepsilon \operatorname{grad} \widetilde{\Phi}_{Q P}+b \widetilde{\Phi}_{Q P}\right)^{T} \operatorname{grad} \widetilde{\Psi}_{P Q} \mathrm{~d} \Omega .
$$

Using (3.3) and $B(-z)=B(z) \exp (z)$, easy calculations show

$$
\left(\varepsilon \operatorname{grad} \widetilde{\Phi}_{Q P}+b \widetilde{\Phi}_{Q P}\right)^{T} \operatorname{grad} \widetilde{\Psi}_{P Q}=-\frac{\varepsilon}{\left[\beta_{P Q}\right]^{2}} B\left(-\frac{\beta_{P Q}}{\varepsilon}\left[e_{P Q}\right]^{T} b\right) .
$$

Therefore, in that case we obtain the statement (see also (4.14)).

Remark 3.3. In [1], where a class of FEMs is studied, the coefficient matrix for the arising system of linear equations has the form

$$
L_{P Q}=\left\{\begin{array}{lll}
-\varepsilon \frac{\gamma_{P Q}}{\beta_{P Q}} K\left(\frac{\beta_{P Q}}{\varepsilon}\left[e_{P Q}\right]^{T} b\right), & \text { if } Q \in N N_{i}(P) & \\
\sum_{R \in N N(P)} \varepsilon \frac{\gamma_{P R}}{\beta_{P R}} K\left(\frac{\beta_{P R}}{\varepsilon}\left[e_{P R}\right]^{T} b\right), & \text { if } Q=P & \text { for } P, Q \in M_{i} \\
0, & \text { otherwise } &
\end{array}\right.
$$

for a suitable function $K$. The choice $K=B$ is possible, but leads to a method which is different to that considered in this paper.

Moreover, we notice that in general the FEMs given in [1] do not conserve the property described in Remark 3.2. 
Corollary 3.2. The problems (2.12) and (3.4) are equivalent, i.e. $u^{V}$ and $u^{E}$ coincide.

Proof. Theorem 3.1 and the relation $b^{E}=\frac{1}{2} b^{V}$ establish the statement.

Remark 3.4. A system of linear equations with the same coefficient matrix as that of the FVM (3.4) arises for the FEM which was studied in [12]. They remarked that such matrices are M-matrices, so that the FVM (3.4) and, because of Corollary 3.2, also the FEM (2.12) have a unique solution. The uniqueness of the solution of problem (2.12) is used later in the application of Lemma 4.1.

\subsection{The notion of convergence for the FVM (3.4)}

We follow the approach given for the Poisson equation in [23].

At first, Corollary 3.2 establishes a bijective correspondence between vectors $u^{V} \in \mathbb{R}^{m}$ solving (3.4) and functions $u_{h} \in V_{h}^{1}$ solving (2.7). Additionally, the interpolation property is satisfied, i.e. $u_{h}(P)=u_{P}^{V}$ for all $P \in M_{i}$.

Now, let a sequence $\left\{M_{n}\right\}$ of sets be given, which satisfy the assumptions of Section 2.1, and let the corresponding maximum stepsize of each set $M_{n}$ be defined by

$$
h_{n}:=h_{n}\left(M_{n}\right):=\max _{d b_{P Q} \in d B_{h}\left(M_{n}\right)} \beta_{P Q} .
$$

Further, let $\left\{u^{V}\left(M_{n}\right)\right\}$ be the sequence of approximate solutions defined by the FVM $(3.4)$, let $\left\{u_{h}\right\}=\left\{u_{h}\left(M_{n}\right)\right\}$ be defined by the FEM (2.7) and let $\|\cdot\|_{h 1}$ be the norm in $V_{h}^{1}=V_{h}^{1}\left(M_{n}\right)$, which is a seminorm on $V \bigoplus V_{h}^{1}$, too. We finally assume that $\lim _{n \rightarrow \infty} h_{n}=0$.

Definition 3.3. The FVM (3.4) is called convergent with respect to $V_{h}^{1}$ and $\|\cdot\|_{h 1}$, iff $\lim _{n \rightarrow \infty}\left\|u-u_{h}\right\|_{h 1}=0$ for the solution $u$ of $(2.1)$.

\section{Application of SeCOnd Strang lemma}

\subsection{A modified second Strang lemma for Petrov-Galerkin FEMs}

A standard approach to prove convergence of nonconforming Galerkin FEMs is the application of the wellknown second Strang lemma (cf. e.g. [6,22]). However, for the nonconforming FEM (2.7), which is a PetrovGalerkin FEM, a modified formulation is necessary as it is given in Lemma 4.1.

Let a Hilbert space $V$, a continuous bilinear form $a: V \times V \rightarrow \mathbb{R}^{1}$ and $d \in V^{\prime}$ be given.

The variational problem:

$$
\text { Find } u \in V \text { such that } a(u, v)=d(v) \quad \forall v \in V
$$

is substituted by the following family of problems:

$$
\text { Find } u_{h} \in V_{h}^{1} \text { such that } a_{h}\left(u_{h}, v_{h}\right)=d_{h}\left(v_{h}\right) \quad \forall v_{h} \in V_{h}^{2} \text {. }
$$

The settings in this context are: $V_{h}^{i}$ with $V_{h}^{i} \not \subset V, i=1,2$, are finite-dimensional Hilbert spaces, whose norms $\|\cdot\|_{h 1}$ and $\|\cdot\|_{h 2}$ are seminorms in $V \bigoplus V_{h}^{1}$ and $V \bigoplus V_{h}^{2}$, respectively; $a_{h}:\left[V \bigoplus V_{h}^{1}\right] \times V_{h}^{2} \rightarrow \mathbb{R}^{1}$ is a bilinear form; $d_{h}$ belongs to the dual space of $V_{h}^{2}$.

Further, let a linear mapping $P_{h}^{2}: V_{h}^{1} \rightarrow V_{h}^{2}$ be given which has the property

$$
P_{h}^{2}\left(v_{h}\right) \neq 0 \quad \forall v_{h} \in V_{h}^{1} \text { with } v_{h} \neq 0 .
$$

Additionally, we assume that the variational problem (4.1) and each problem (4.2) have a unique solution $u$ and $u_{h}$, respectively. 
Lemma 4.1. Assume that the bilinear form $a_{h}$ satisfies

$$
\left|a_{h}\left(v, w_{h}\right)\right| \leq c\|v\|_{h 1}\left\|w_{h}\right\|_{h 2} \quad \forall v \in V \oplus V_{h}^{1}, w_{h} \in V_{h}^{2}
$$

and

$$
a_{h}\left(v_{h}, P_{h}^{2}\left(v_{h}\right)\right) \geq \alpha\left\|P_{h}^{2}\left(v_{h}\right)\right\|_{h 2}^{2} \quad \forall v_{h} \in V_{h}^{1}
$$

with some positive constants $c$ and $\alpha$ independent of $h$.

Then there exist positive constants $C_{1}$ and $C_{2}$ independent of $h$ such that

$$
\left\|u-P_{h}^{2}\left(u_{h}\right)\right\|_{h 2} \leq \inf _{v_{h} \in V_{h}^{1}}\left\{\left\|u-P_{h}^{2}\left(v_{h}\right)\right\|_{h 2}+C_{1}\left\|u-v_{h}\right\|_{h 1}\right\}+C_{2} \sup _{w_{h} \in V_{h}^{2}} \frac{\left|a_{h}\left(u, w_{h}\right)-d_{h}\left(w_{h}\right)\right|}{\left\|w_{h}\right\|_{h 2}} .
$$

Proof. The proof is substantially the same as for the standard second Strang lemma. Therefore, we refer to [24], where the details are given.

Remark 4.1. In [12] a different approach is used to prove the convergence of Petrov-Galerkin FEMs. By a linear mapping the Petrov-Galerkin FEM was transformed into an equivalent Galerkin FEM, which was analyzed by the standard second Strang lemma. But an advantage of Lemma 4.1 is that in contrast to the standard second Strang lemma two different Hilbert spaces can be used, one for the ansatz functions and another one for the test functions.

In the application of the second Strang lemma the following inequality is often used to estimate the terms on the right-hand side

$$
\inf _{v_{h} \in V_{h}}\left\|u-v_{h}\right\|_{h} \leq\|u-\Pi(u)\|_{h}
$$

where $\Pi$ denotes a linear mapping with $\Pi: V \rightarrow V_{h}$ or $\Pi: W \subset V \rightarrow V_{h}$.

To extend this approach to the term

$$
\inf _{v_{h} \in V_{h}^{1}}\left\{\left\|u-P_{h}^{2}\left(v_{h}\right)\right\|_{h 2}+C_{1}\left\|u-v_{h}\right\|_{h 1}\right\}
$$

we assume that a subspace $W \subset V$ and a linear mapping $P_{h}^{1}: W \rightarrow V_{h}^{1}$ are given.

Corollary 4.2. Let be $u \in W$ and let the assumptions of Lemma 4.1 be satisfied.

Then there exist positive constants $C_{1}$ and $C_{2}$ independent of $h$ such that

$$
\left\|u-P_{h}^{2}\left(u_{h}\right)\right\|_{h 2} \leq\left\|u-P_{h}^{2}\left(P_{h}^{1}(u)\right)\right\|_{h 2}+C_{1}\left\|u-P_{h}^{1}(u)\right\|_{h 1}+C_{2} \sup _{w_{h} \in V_{h}^{2}} \frac{\left|a_{h}\left(u, w_{h}\right)-d_{h}\left(w_{h}\right)\right|}{\left\|w_{h}\right\|_{h 2}} .
$$

\subsection{The application to the problems (2.1) and (2.7)}

We start by defining $P_{h}^{2}$ and $P_{h}^{1}$.

If the exact solution of the continuous problem is sufficiently smooth, then in (4.6) the mapping $\Pi$ is usually chosen as the $V_{h}$-interpolation operator. Following this approach we assume that for the solution $u$ of (2.1) there holds $u \in W$ with

$$
W:=H^{2}(\Omega) \cap V=H^{2}(\Omega) \cap H_{0}^{1}(\Omega),
$$


and we define the linear mappings $P_{h}^{2}: W \bigoplus V_{h}^{1} \rightarrow V_{h}^{2}$ and $P_{h}^{1}: W \bigoplus V_{h}^{2} \rightarrow V_{h}^{1}$ by

$$
P_{h}^{2}\left(v+v_{h}\right):=\sum_{P \in M_{i}}\left[v(P)+v_{h}(P)\right] \Psi_{P} \quad \forall v \in W, v_{h}=\sum_{P \in M_{i}} v_{h}(P) \Phi_{P} \in V_{h}^{1}
$$

and

$$
P_{h}^{1}\left(v+v_{h}\right):=\sum_{P \in M_{i}}\left[v(P)+v_{h}(P)\right] \Phi_{P} \quad \forall v \in W, v_{h}=\sum_{P \in M_{i}} v_{h}(P) \Psi_{P} \in V_{h}^{2},
$$

where $\Phi$ and $\Psi$ are the nodal basis functions in $V_{h}^{1}$ and $V_{h}^{2}$, respectively.

Obviously, the mapping $P_{h}^{2}$ satisfies the property (4.3), and, especially, it is such that:

$$
P_{h}^{2}\left(P_{h}^{1}(v)\right)=P_{h}^{2}(v) \quad \forall v \in W .
$$

Further, we define norms $\|\cdot\|_{h 1}$ in $V_{h}^{1}$ and $\|\cdot\|_{h 2}$ in $V_{h}^{2}$ by

$$
\|v\|_{h 1}:=\left\{\frac{1}{\varepsilon} \sum_{d b_{P Q} \in d B_{h}} \iint_{d b_{P Q}}\left(\left[e_{P Q}\right]^{T}[\varepsilon \operatorname{grad} v+b v]\right)^{2} \mathrm{~d} \Omega\right\}^{\frac{1}{2}}
$$

and

$$
\|v\|_{h 2}:=\left\{\varepsilon \sum_{d b_{P Q} \in d B_{h}} \iint_{d b_{P Q}}\left(\left[e_{P Q}\right]^{T} \operatorname{grad} v\right)^{2} \mathrm{~d} \Omega\right\}^{\frac{1}{2}} .
$$

Obviously, $V_{h}^{1}$ and $V_{h}^{2}$ are Hilbert spaces, and $\|\cdot\|_{h 1}$ and $\|\cdot\|_{h 2}$ are also seminorms on $V \bigoplus V_{h}^{1}$ and $V \bigoplus V_{h}^{2}$, respectively.

Remark 4.2. Our convergence result given in Section 5 is presented in the form of an estimate for $\left\|u-P_{h}^{2}\left(u_{h}\right)\right\|_{h 2}$. This does not imply that $u_{h}$ converges to $u$ in any norm in $V$. However, our convergence result is stronger than that obtained by Lazarov et al. [11], by Eymard et al. [7] or by Miller and Wang [12]. Because of (4.15), e.g. the estimates by Lazarov et al. [11] and by Eymard et al. [7] are only estimates for $\left\|P_{h}^{2}(u)-P_{h}^{2}\left(u_{h}\right)\right\|_{h 2}$.

Remark 4.3. In the following proofs, we simplify some notations. Sometimes, $\beta_{P Q}$ and $\gamma_{P Q}$ are replaced by $\beta$ and $\gamma$, respectively. Further, $e$ and $t$ are used, which on the dual Voronoi box $d b_{P Q}$ have the meanings $e_{P Q}$ defined by (2.4) and $t_{P Q}$ defined by (6.15), respectively.

Theorem 4.3. Let $\|\cdot\|_{h 2}$ and $a_{h}$ be defined by (4.12b) and (2.8), respectively.

Then (4.4b) holds with $\alpha=1$.

Proof. Obviously, in $\operatorname{Int}\left(d b_{P Q}\right)$ it holds

$$
\operatorname{grad} w_{h}=\frac{w_{h}(Q)-w_{h}(P)}{\beta_{P Q}} e_{P Q} \quad \forall w_{h} \in V_{h}^{2} .
$$

Hence, because of (4.9) for each $v_{h} \in V_{h}^{1}$ we obtain

$$
a_{h}\left(v_{h}, P_{h}^{2}\left(v_{h}\right)\right)=\sum_{d b_{P Q} \in d B_{h}} \frac{1}{\beta}\left[v_{h}(Q)-v_{h}(P)\right] \iint_{d b_{P Q}} e^{T}\left[\varepsilon \operatorname{grad} v_{h}+b v_{h}\right] \mathrm{d} \Omega .
$$


Using

$$
\iint_{d b_{P Q}} \mathrm{~d} \Omega=\frac{1}{2} \beta_{P Q} \gamma_{P Q}
$$

easy calculations show (see also proof of Th. 3.1) that

$$
a_{h}\left(v_{h}, P_{h}^{2}\left(v_{h}\right)\right)=\frac{\varepsilon}{2} \sum_{d b_{P Q} \in d B_{h}} \frac{\gamma}{\beta}\left[B\left(\frac{\beta}{\varepsilon} e^{T} b\right) v_{h}(P)-B\left(-\frac{\beta}{\varepsilon} e^{T} b\right) v_{h}(Q)\right]\left[v_{h}(P)-v_{h}(Q)\right] .
$$

The proof of Theorem 4.1 in [12] takes advantage of the equality

$$
\begin{aligned}
2\left[v_{h}(P)-\exp (z) v_{h}(Q)\right]\left[v_{h}(P)-v_{h}(Q)\right]= & {[1+\exp (z)]\left[v_{h}(P)-v_{h}(Q)\right]^{2} } \\
& +[1-\exp (z)]\left\{\left[v_{h}(P)\right]^{2}-\left[v_{h}(Q)\right]^{2}\right\} \quad \forall z \in \mathbb{R}^{1} .
\end{aligned}
$$

Using $B(-z)=B(z) \exp (z)$ and $B(z)[1-\exp (z)]=-z$ this yields

$$
\begin{aligned}
2\left[B(z) v_{h}(P)-B(-z) v_{h}(Q)\right]\left[v_{h}(P)-v_{h}(Q)\right]= & B(z)[1+\exp (z)]\left[v_{h}(P)-v_{h}(Q)\right]^{2} \\
& -z\left\{\left[v_{h}(P)\right]^{2}-\left[v_{h}(Q)\right]^{2}\right\} \quad \forall z \in \mathbb{R}^{1} .
\end{aligned}
$$

Hence, we deduce that

$$
\begin{aligned}
a_{h}\left(v_{h}, P_{h}^{2}\left(v_{h}\right)\right)= & \frac{\varepsilon}{4} \sum_{d b_{P Q} \in d B_{h}} B\left(\frac{\beta}{\varepsilon} e^{T} b\right)\left[1+\exp \left(\frac{\beta}{\varepsilon} e^{T} b\right)\right] \frac{\gamma}{\beta}\left[v_{h}(P)-v_{h}(Q)\right]^{2} \\
& -\frac{1}{4} \sum_{d b_{P Q} \in d B_{h}} \gamma e^{T} b\left\{\left[v_{h}(P)\right]^{2}-\left[v_{h}(Q)\right]^{2}\right\}
\end{aligned}
$$

Since $e_{P Q}=-e_{Q P}$, for all $v_{h} \in V_{h}^{1}$ it follows that

$$
\sum_{d b_{P Q} \in d B_{h}} \gamma_{P Q}\left[e_{P Q}\right]^{T} b\left\{\left[v_{h}(P)\right]^{2}-\left[v_{h}(Q)\right]^{2}\right\}=\sum_{P \in M_{i}}\left\{\left[v_{h}(P)\right]^{2} \sum_{Q \in N N(P)} \gamma_{P Q}\left[e_{P Q}\right]^{T} b\right\} .
$$

Using (A), the fact that $\left.n\left(b_{P}\right)\right|_{b_{P} \cap b_{Q}}=e_{P Q}$ for the outer normal direction $n\left(b_{P}\right)$ of the Voronoi box $b_{P}$ and recalling that $b$ is constant, we have

$$
\sum_{Q \in N N(P)} \gamma_{P Q}\left[e_{P Q}\right]^{T} b=\oint_{\partial b_{P}}\left[n\left(b_{P}\right)\right]^{T} b \mathrm{~d} \Gamma=\iint_{b_{P}} \operatorname{div} b \mathrm{~d} \Omega=0 \quad \forall P \in M_{i} .
$$

This yields

$$
a_{h}\left(v_{h}, P_{h}^{2}\left(v_{h}\right)\right)=\frac{\varepsilon}{4} \sum_{d b_{P Q} \in d B_{h}} B\left(\frac{\beta}{\varepsilon} e^{T} b\right)\left[1+\exp \left(\frac{\beta}{\varepsilon} e^{T} b\right)\right] \frac{\gamma}{\beta}\left[v_{h}(P)-v_{h}(Q)\right]^{2} .
$$

Further, because of (4.13) and (4.14) we obtain

$$
\left\|w_{h}\right\|_{h 2}^{2}=\frac{\varepsilon}{2} \sum_{d b_{P Q} \in d B_{h}} \frac{\gamma_{P Q}}{\beta_{P Q}}\left[w_{h}(P)-w_{h}(Q)\right]^{2} \quad \forall w_{h} \in V_{h}^{2} .
$$


Observing that

$$
B(z)[1+\exp (z)] \geq 2 \quad \forall z \in \mathbb{R}^{1}
$$

(which can be proved by easy calculation), the statement follows by (4.15) and (4.9).

Remark 4.4. In [12] the inequality $B(z)[1+\exp (z)] \geq|z| \forall z \in \mathbb{R}^{1}$ is applied instead of (4.16) to estimate

$$
\frac{\varepsilon}{4} \sum_{d b_{P Q} \in d B_{h}} B\left(\frac{\beta}{\varepsilon} e^{T} b\right)\left[1+\exp \left(\frac{\beta}{\varepsilon} e^{T} b\right)\right] \frac{\gamma}{\beta}\left[v_{h}(P)-v_{h}(Q)\right]^{2}
$$

Moreover, two restrictive assumptions are used in [12], namely, a quasi-uniform mesh and the assumption

$$
\left|\left[e_{P Q}\right]^{T} b\right| \geq b_{0}>0 \quad \forall d b_{P Q} \in d B_{h}
$$

Theorem 4.4. Let $\|\cdot\|_{h 1},\|\cdot\|_{h 2}$ and $a_{h}$ be defined by (4.12) and (2.8), respectively.

Then (4.4a) holds with $c=1$.

Proof. From (4.13) we infer that

$$
\begin{aligned}
\left|a_{h}\left(v, w_{h}\right)\right| & \leq \sum_{d b_{P Q} \in d B_{h}} \frac{1}{\beta}\left|w_{h}(Q)-w_{h}(P)\right|\left|\iint_{d b_{P Q}} e^{T}[\varepsilon \operatorname{grad} v+b v] \mathrm{d} \Omega\right| \\
& \leq\left\{\frac{2}{\varepsilon} \sum_{d b_{P Q} \in d B_{h}} \frac{1}{\beta \gamma}\left(\iint_{d b_{P Q}} e^{T}[\varepsilon \operatorname{grad} v+b v] \mathrm{d} \Omega\right)^{2}\right\}\left\{\frac{\varepsilon}{2} \sum_{d b_{P Q} \in d B_{h}} \frac{\gamma}{\beta}\left[w_{h}(P)-w_{h}(Q)\right]^{2}\right\}^{\frac{1}{2}} \cdot
\end{aligned}
$$

By (4.14) we deduce that

$$
\left(\iint_{d b_{P Q}} e^{T}[\varepsilon \operatorname{grad} v+b v] \mathrm{d} \Omega\right)^{2} \leq\left\{\frac{1}{2} \beta \gamma\right\}\left\{\iint_{d b_{P Q}}\left(e^{T}[\varepsilon \operatorname{grad} v+b v]\right)^{2} \mathrm{~d} \Omega\right\}
$$

Using (4.15) and (4.12) concludes the proof of the theorem.

Corollary 4.5. Let $u \in H^{2}(\Omega) \cap H_{0}^{1}(\Omega)$ be the solution of the continuous problem (2.1) and let $u_{h} \in V_{h}^{2}$ be a solution of the FEM (2.7).

Then, with $\|\cdot\|_{h 1},\|\cdot\|_{h 2}, P_{h}^{1}$ and $P_{h}^{2}$ defined by (4.12), (4.10) and (4.9), respectively, it holds

$$
\left\|u-P_{h}^{2}\left(u_{h}\right)\right\|_{h 2} \leq\left\|u-P_{h}^{2}(u)\right\|_{h 2}+\left\|u-P_{h}^{1}(u)\right\|_{h 1}+\sup _{w_{h} \in V_{h}^{2}} \frac{\left|a_{h}\left(u, w_{h}\right)-d_{h}\left(w_{h}\right)\right|}{\left\|w_{h}\right\|_{h 2}}
$$

Proof. The statement is an easy consequence of (4.11), Corollary 4.2 and Theorems 4.3 and 4.4. 


\section{The COnVERGEnCE RESUlt}

Corollaries 4.5, 7.5 and 7.9 result in:

Theorem 5.1. For a sequence $\left\{M_{n}\right\}$ of sets satisfying the assumptions of Section 2.1 let $\left\{u_{h}\right\}:=\left\{u_{h}\left(M_{n}\right)\right\}$ be the corresponding sequence of FEM solutions defined by (2.7).

Additionally, let (6.1) and (6.2) be satisfied.

Further, we assume that $\lim _{n \rightarrow \infty} h_{n}=0$ with $h_{n}$ defined by (3.5).

If $u \in H^{2}(\Omega) \cap H_{0}^{1}(\Omega)$ is the solution of (2.1), there exist positive constants $C_{1}$ and $C_{2}$ independent of $n$, $\varepsilon$ and $b$, such that

$$
\left\|u-P_{h}^{2}\left(u_{h}\right)\right\|_{h 2} \leq \sqrt{\varepsilon}\left(C_{1} h_{n}|u|_{2, \Omega}+C_{2}\left\{\sum_{d b_{P Q} \in d B_{h}\left(M_{n}\right)}\left[\beta_{P Q}\right]^{2}|u|_{\varepsilon, 2, d b_{P Q}}^{2}\right\}^{\frac{1}{2}}\right)
$$

Thereby, $P_{h}^{2},\|\cdot\|_{h 2}$ and $|\cdot|_{\varepsilon, 2, d b_{P Q}}$ are defined by (4.9), (4.12b) and (6.14), respectively.

Because of (6.18a), Theorem 5.1 states the linear convergence of the FEM (2.7) for each fixed $\varepsilon$.

Moreover, using Corollary 3.2 and Definition 3.3 the estimate (5.1) as well as the resulting convergence properties of the FEM (2.7) can be extended to the FVM (3.4).

Remark 5.1. In [25] a new FEM is presented for convection-diffusion problems. This method uses Delaunay triangulations and conforming linear elements.

In the two-dimensional case and for the problem (1.1), the coefficient matrix of the arising system of linear equations coincides with that of the FVM (3.4) and the estimate obtained by Xu and Zikatanov [25] has the form

$$
\left\|\Pi_{n}(u)-u_{h}\right\|_{1, \Omega} \leq C h_{n}\left\{\sum_{t \in T_{n}}|\varepsilon \operatorname{grad} u+b u|_{1, t}^{2}\right\}^{\frac{1}{2}}
$$

where $\left\{T_{n}\right\}$ is a sequence of Delaunay triangulations and $\Pi_{n}$ denotes the interpolation operator. Since the FEM in [25] is a conforming one, they use other techniques to prove their results.

At first sight, the estimate (5.2) seems to be better than (5.1) as far as the dependence on $\varepsilon$ is concerned. However, this is not clear, since the constant $\mathrm{C}$ in (5.2) may depend on $\varepsilon$.

In [8] a new FEM for convection-diffusion problems which is based on Delaunay triangulations and conforming linear elements is presented and analyzed, too. Under the additional assumption of a quasi-uniform triangulation, they can prove estimates which are comparable to (5.2).

\section{Preliminaries}

\subsection{Assumptions concerning the partition}

To obtain estimates for the terms on the right-hand side of (4.17) we make the following assumptions:

There exist positive constants $c_{i}, i=1,2,3$, such that

$$
c_{1} \leq \frac{\gamma_{P Q}}{\beta_{P Q}} \leq c_{2} \quad \text { and } \quad \frac{\left|E_{1}(P, Q)-P\right|}{\beta_{P Q}} \leq c_{3} \quad \forall d b_{P Q} \in d B_{h}
$$

(6.1) guarantees that for a sequence of dual box partitions all constants which depend on $d b_{P Q}$ have an upper bound independent of $d b_{P Q}$ (see e.g. proof of Corollary 7.5). 
Remark 6.1. Assumption (6.1) can be weakened at the price of a more complicated mapping $F$ in (6.3) (cf. [23]). In order to simplify the presentation we restrict ourselves to the simple mapping.

We also notice that (6.1) is nearly the same as the minimal angle condition for a sequence of corresponding Delaunay triangulations (see again [23]).

To obtain estimates independent of $\varepsilon$ we additionally assume that:

There exists a positive constant $C$ such that $\beta_{P Q}\left|\left[e_{P Q}\right]^{T} b\right| \leq C \varepsilon \quad \forall d b_{P Q} \in d B_{h}$.

Remark 6.2. Obviously, for each fixed $\varepsilon$ the assumption (6.2) is not restrictive. However, in the case of singularly perturbed problems, where the parameter $\varepsilon$ is small, it requires meshes of the size $h=O(\varepsilon)$ in the whole domain.

\subsection{The affine-equivalent reference element $\hat{d b}_{P Q}$}

To obtain uniformly bounded constants in the error estimates, for each $d b_{P Q}$ we define bijective affine mappings $F: \mathbb{R}^{2} \rightarrow \mathbb{R}^{2}$ and $\hat{F}: \mathbb{R}^{2} \rightarrow \mathbb{R}^{2}$ by

$$
Z=F(\hat{Z}):=G \hat{Z}+g \quad \text { and } \quad \hat{Z}=\hat{F}(Z):=F^{-1}(Z) .
$$

The matrix $G$ and the vector $g$ are given by

$$
G:=\frac{\beta_{P Q}}{2}\left(\begin{array}{rr}
\cos \phi & -\sin \phi \\
\sin \phi & \cos \phi
\end{array}\right)
$$

and $g:=\frac{1}{2}(P+Q)$, respectively, with the angle $\phi:=\Varangle\left(Q-P, E_{y}\right)$, where $E_{y}=(0,1)^{T}$ denotes the unit vector in the $y$-direction.

Now, the reference element $\hat{d} b_{P Q}$, which corresponds to the Voronoi box $d b_{P Q}$, is defined by $\hat{d} b_{P Q}:=$ $\hat{F}\left(d b_{P Q}\right)$. In the following, $d b_{P Q}$ and $\hat{d} b_{P Q}$ are called affine-equivalent domains, too.

There holds $\hat{F}\left(E_{i}\right)=:\left(\hat{x}_{i}, 0\right)^{T}, i=1,2$, as well as

$$
\hat{\gamma}_{P Q}:=\hat{x}_{2}-\hat{x}_{1}=2 \frac{\gamma_{P Q}}{\beta_{P Q}},
$$

where $E_{1}=E_{1}(P, Q)$ and $E_{2}=E_{2}(P, Q)$ are the points defined as in Section 2.1, but henceforth such that $\Varangle\left(E_{2} P E_{1}\right)>0$.

We have that

$$
\operatorname{det}(G)=\frac{1}{4}\left[\beta_{P Q}\right]^{2}
$$

and that the spectral norms $|$.$| of G$ and $G^{-1}$, respectively, can be estimated by

$$
|G| \leq \frac{1}{2} \beta_{P Q} \quad \text { and } \quad\left|G^{-1}\right| \leq \frac{1}{2} \frac{1}{\beta_{P Q}} .
$$

These affine transformations also yield an unique correspondence between functions $v: d b_{P Q} \rightarrow \mathbb{R}^{1}$ and $\hat{v}: \hat{d} b_{P Q} \rightarrow \mathbb{R}^{1}$, which is defined by

$$
\hat{v}(\hat{Z})=v(Z) \quad \text { with } \quad Z=F(\hat{Z}) .
$$


In particular,

$$
v \in L K_{\varepsilon}(P, Q) \Longleftrightarrow \hat{v} \in L \hat{K}_{\varepsilon}(P, Q) \quad \text { and } \quad v \in \widetilde{P}(P, Q) \Longleftrightarrow \hat{v} \in \hat{\widetilde{P}}:=\operatorname{span}\{1, \hat{y}\}
$$

with

$$
L \hat{K}_{\varepsilon}(P, Q):= \begin{cases}\operatorname{span}\left\{1, F_{3, \varepsilon}(\hat{y})\right\}, & \text { if }\left[e_{P Q}\right]^{T} b \neq 0 \\ \operatorname{span}\{1, \hat{y}\}, & \text { if }\left[e_{P Q}\right]^{T} b=0\end{cases}
$$

and

$$
F_{3, \varepsilon}(\hat{y}):=\exp \left(-\hat{y} \frac{\beta_{P Q}}{2 \varepsilon}\left[e_{P Q}\right]^{T} b\right) .
$$

Further, we obtain e.g. $\left(\right.$ with $\left.\hat{Z}=(\hat{x}, \hat{y})^{T}\right)$

$$
\hat{\widetilde{\Phi}}_{P Q}(\hat{Z})= \begin{cases}\frac{1}{\exp \left(\frac{\beta_{P Q}}{\varepsilon}\left[e_{P Q}\right]^{T} b\right)-1}\left[-1+\exp \left(\frac{\beta_{P Q}}{2 \varepsilon}\left[e_{P Q}\right]^{T} b\right) F_{3, \varepsilon}(\hat{y})\right], & \text { if }\left[e_{P Q}\right]^{T} b \neq 0 \\ \frac{1-\hat{y}}{2}, & \text { if }\left[e_{P Q}\right]^{T} b=0 .\end{cases}
$$

We conclude this subsection by noting that

$$
\iint_{\hat{d} b_{P Q} \cap \hat{b}_{P}} \mathrm{~d} \hat{\Omega}=\iint_{\hat{d} b_{P Q} \cap \hat{b}_{Q}} \mathrm{~d} \hat{\Omega}=\frac{1}{2} \iint_{\hat{d} b_{P Q}} \mathrm{~d} \hat{\Omega}=\frac{\gamma_{P Q}}{\beta_{P Q}} \quad \text { and } \quad \int_{\hat{d} b_{P Q}^{12}} \mathrm{~d} \hat{\Gamma}=2 \frac{\gamma_{P Q}}{\beta_{P Q}}
$$

with $\hat{b}_{P}:=\hat{F}\left(b_{P}\right), \hat{b}_{Q}:=\hat{F}\left(b_{Q}\right)$,

$$
d b_{P Q}^{12}:=b_{P} \cap b_{Q}
$$

and $\hat{d b} b_{P Q}^{12}:=\hat{F}\left(d b_{P Q}^{12}\right)$.

\subsection{Suitable Hilbert spaces and relations with Sobolev spaces}

For our purpose, it is useful to substitute the Sobolev space $H^{1}\left(d b_{P Q}\right)$ by the space $\tilde{H}^{1}\left(d b_{P Q}\right)$ defined by

$$
\tilde{H}^{1}\left(d b_{P Q}\right):=\left\{q \in L_{2}\left(d b_{P Q}\right):\left[e_{P Q}\right]^{T} \operatorname{grad} q \in L_{2}\left(d b_{P Q}\right)\right\},
$$

which is a Hilbert space with respect to the norm and the seminorm

$$
\|q\|_{\tilde{H}^{1}\left(d b_{P Q}\right)}:=\left\{\|q\|_{0, d b_{P Q}}^{2}+|q|_{\tilde{H}^{1}\left(d b_{P Q}\right)}^{2}\right\}^{\frac{1}{2}} \quad \text { and } \quad|q|_{\tilde{H}^{1}\left(d b_{P Q}\right)}:=\left\|\left[e_{P Q}\right]^{T} \operatorname{grad} q\right\|_{0, d b_{P Q}},
$$

respectively (cf. [23]). Besides, we will also use some other norms and seminorms in $\tilde{H}^{1}\left(d b_{P Q}\right)$ and $H^{2}\left(d b_{P Q}\right)$, which depend on $\varepsilon$ and $\left[e_{P Q}\right]^{T} b$. Those ones are defined by

$$
\begin{gathered}
\|q\|_{\varepsilon, \tilde{H}^{1}\left(d b_{P Q}\right)}:=\left\{\|q\|_{0, d b_{P Q}}^{2}+|q|_{\varepsilon, \tilde{H}^{1}\left(d b_{P Q}\right)}^{2}\right\}^{\frac{1}{2}}, \quad\|q\|_{\varepsilon, 2, d b_{P Q}}:=\left\{\|q\|_{1, d b_{P Q}}^{2}+|q|_{\varepsilon, 2, d b_{P Q}}^{2}\right\}^{\frac{1}{2}}, \\
|q|_{\varepsilon, \tilde{H}^{1}\left(d b_{P Q}\right)}:=\left\|\left[e_{P Q}\right]^{T}\left[\operatorname{grad} q+\frac{1}{\varepsilon} b q\right]\right\|_{0, d b_{P Q}}
\end{gathered}
$$


and

$$
\begin{aligned}
|q|_{\varepsilon, 2, d b_{P Q}}:= & {\left[\left\|\left[t_{P Q}\right]^{T} H_{q} t_{P Q}\right\|_{0, d b_{P Q}}^{2}+2\left\|\left[e_{P Q}\right]^{T} H_{q} t_{P Q}+\frac{\left[e_{P Q}\right]^{T} b}{\varepsilon}\left[t_{P Q}\right]^{T} \operatorname{grad} q\right\|_{0, d b_{P Q}}^{2}\right.} \\
& \left.+\left\|\left[e_{P Q}\right]^{T} H_{q} e_{P Q}+\frac{\left[e_{P Q}\right]^{T} b}{\varepsilon}\left[e_{P Q}\right]^{T} \operatorname{grad} q\right\|_{0, d b_{P Q}}^{2}\right]^{\frac{1}{2}} \cdot
\end{aligned}
$$

Thereby, $H_{q}$ denotes the matrix of weak second derivatives of $q$ and the vector $t_{P Q}$ is defined by

$$
t_{P Q}:=\frac{E_{2}(P, Q)-E_{1}(P, Q)}{\left|E_{2}(P, Q)-E_{1}(P, Q)\right|}=\frac{1}{\beta_{P Q}}\left(y_{Q}-y_{P}, x_{P}-x_{Q}\right)^{T},
$$

such that there holds $\left[e_{P Q}\right]^{T} t_{P Q}=0$.

Obviously, $\|\cdot\|_{\varepsilon, \tilde{H}^{1}\left(d b_{P Q}\right)}$ and $\|\cdot\|_{\varepsilon, 2, d b_{P Q}}$ are based on scalar products, such that $\tilde{H}^{1}\left(d b_{P Q}\right)$ and $H^{2}\left(d b_{P Q}\right)$ with these norms are Hilbert spaces, too.

Remark 6.3. In the case $\left[e_{P Q}\right]^{T} b=0$ the seminorms defined by (6.13) and (6.14) are independent of $\varepsilon$. Then there even hold $\|q\|_{\varepsilon, \tilde{H}^{1}\left(d b_{P Q}\right)}=\|q\|_{\tilde{H}^{1}\left(d b_{P Q}\right)}$ for all $q \in \tilde{H}^{1}\left(d b_{P Q}\right)$ and $\|q\|_{\varepsilon, 2, d b_{P Q}}=\|q\|_{2, d b_{P Q}}$ for all $q \in H^{2}\left(d b_{P Q}\right)$.

Since we want to follow standard techniques for error estimations it is necessary to introduce corresponding spaces, seminorms and norms on the reference element $\hat{d}_{P Q}$.

The space $\tilde{H}^{1}\left(\hat{d}_{P Q}\right)$ is defined by

$$
\tilde{H}^{1}\left(\hat{d}_{P Q}\right):=\left\{\hat{q} \in L_{2}\left(\hat{d}_{P Q}\right): \hat{q}_{\hat{y}} \in L_{2}\left(\hat{d}_{P Q}\right)\right\}
$$

the norms and seminorms in $\tilde{H}^{1}\left(\hat{d}_{P Q}\right)$ and $H^{2}\left(\hat{d} b_{P Q}\right)$, respectively, are defined by

$$
\begin{array}{r}
\|\hat{q}\|_{\varepsilon, \tilde{H}^{1}\left(\hat{d} b_{P Q}\right)}:=\left\{\|\hat{q}\|_{0, \hat{d} b_{P Q}}^{2}+|\hat{q}|_{\varepsilon, \tilde{H}^{1}\left(\hat{d} b_{P Q}\right)}^{2}\right\}^{\frac{1}{2}}, \quad\|\hat{q}\|_{\varepsilon, 2, \hat{d} b_{P Q}}:=\left\{\|\hat{q}\|_{1, \hat{d} b_{P Q}}^{2}+|\hat{q}|_{\varepsilon, 2, \hat{d} b_{P Q}}^{2}\right\}^{\frac{1}{2}}, \\
|\hat{q}|_{\varepsilon, \tilde{H}^{1}\left(\hat{d} b_{P Q}\right)}:=\left\|\hat{q}_{\hat{y}}+\hat{q} \frac{\beta P Q}{2 \varepsilon}\left[e_{P Q}\right]^{T} b\right\|_{0, \hat{d} b_{P Q}}
\end{array}
$$

and

$$
|\hat{q}|_{\varepsilon, 2, \hat{d} b_{P Q}}:=\left[\left\|\hat{q}_{\hat{x} \hat{x}}\right\|_{0, \hat{d} b_{P Q}}^{2}+2\left\|\hat{q}_{\hat{x} \hat{y}}+\hat{q}_{\hat{x}} \frac{\beta_{P Q}}{2 \varepsilon}\left[e_{P Q}\right]^{T} b\right\|_{0, \hat{d} b_{P Q}}^{2}+\left\|\hat{q}_{\hat{y} \hat{y}}+\hat{q}_{\hat{y}} \frac{\beta_{P Q}}{2 \varepsilon}\left[e_{P Q}\right]^{T} b\right\|_{0, \hat{d} b_{P Q}}^{2}\right]^{\frac{1}{2}} .
$$

Lemma 6.1. It holds

$$
\begin{aligned}
& |v|_{\varepsilon, 2, d b_{P Q}}^{2} \leq 2|v|_{2, d b_{P Q}}^{2}+4\left[\frac{\left[e_{P Q}\right]^{T} b}{\varepsilon}\right]^{2}|v|_{1, d b_{P Q}}^{2} \quad \forall v \in H^{2}\left(d b_{P Q}\right), \\
& |\hat{v}|_{\varepsilon, 2, \hat{d} b_{P Q}}^{2} \leq 2|\hat{v}|_{2, \hat{d} b_{P Q}}^{2}+\left[\frac{\beta_{P Q}}{\varepsilon}\left[e_{P Q}\right]^{T} b\right]^{2}|\hat{v}|_{1, \hat{d} b_{P Q}}^{2} \quad \forall \hat{v} \in H^{2}\left(\hat{d} b_{P Q}\right),
\end{aligned}
$$

and

$$
|\hat{v}|_{\varepsilon, \tilde{H}^{1}\left(\hat{d} b_{P Q}\right)}^{2} \leq 2|\hat{v}|_{1, \hat{d} b_{P Q}}^{2}+\frac{1}{2}\left[\frac{\beta_{P Q}}{\varepsilon}\left[e_{P Q}\right]^{T} b\right]^{2}\|\hat{v}\|_{0, \hat{d} b_{P Q}}^{2} \quad \forall \hat{v} \in H^{1}\left(\hat{d} b_{P Q}\right)
$$


Proof. Due to inequalities like

$$
\left(e^{T} H_{v} t+\left[\frac{e^{T} b}{\varepsilon}\right] t^{T} \operatorname{grad} v\right)^{2} \leq 2\left(e^{T} H_{v} t\right)^{2}+2\left[\frac{e^{T} b}{\varepsilon}\right]^{2}\left(t^{T} \operatorname{grad} v\right)^{2}
$$

we obtain

$$
\begin{aligned}
& \left(t^{T} H_{v} t\right)^{2}+2\left(e^{T} H_{v} t+\left[\frac{e^{T} b}{\varepsilon}\right] t^{T} \operatorname{grad} v\right)^{2}+\left(e^{T} H_{v} e+\left[\frac{e^{T} b}{\varepsilon}\right] e^{T} \operatorname{grad} v\right)^{2} \\
& \leq 2\left\{\left(t^{T} H_{v} t\right)^{2}+2\left(e^{T} H_{v} t\right)^{2}+\left(e^{T} H_{v} e\right)^{2}\right\}+4\left[\frac{e^{T} b}{\varepsilon}\right]^{2}\left\{\left(t^{T} \operatorname{grad} v\right)^{2}+\left(e^{T} \operatorname{grad} v\right)^{2}\right\}
\end{aligned}
$$

Using (2.4) and (6.15) easy calculations show that

$$
\left(t^{T} \operatorname{grad} v\right)^{2}+\left(e^{T} \operatorname{grad} v\right)^{2}=v_{x}^{2}+v_{y}^{2} \quad \text { and } \quad\left(t^{T} H_{v} t\right)^{2}+2\left(e^{T} H_{v} t\right)^{2}+\left(e^{T} H_{v} e\right)^{2}=v_{x x}^{2}+2 v_{x y}^{2}+v_{y y}^{2}
$$

which proves $(6.18 \mathrm{a})$. In the same way $(6.18 \mathrm{~b})$ and $(6.18 \mathrm{c})$ can be proved.

Lemma 6.2. Assuming (6.2), then the norms $\|\cdot\|_{\varepsilon, 2, \hat{d} b_{P Q}}$ and $\|\cdot\|_{2, \hat{d} b_{P Q}}$ are equivalent independently of $\varepsilon$ and $\beta_{P Q}$.

Proof. To obtain the desired norm equivalence we have to show that

$$
C_{1}\|\hat{v}\|_{\varepsilon, 2, \hat{d} b_{P Q}} \leq\|\hat{v}\|_{2, \hat{d} b_{P Q}} \leq C_{2}\|\hat{v}\|_{\varepsilon, 2, \hat{d} b_{P Q}} \quad \forall \hat{v} \in H^{2}\left(\hat{d} b_{P Q}\right)
$$

for some constants $C_{i}=C_{i}\left(\hat{d b}_{P Q}\right), i=1,2$, independent of $\varepsilon$ and $\beta_{P Q}$.

The left inequality is an easy consequence of (6.18b) and (6.2).

To prove the rest, we use inequalities like the following one

$$
\left(\hat{v}_{\hat{y} \hat{y}}+\hat{v}_{\hat{y}} \frac{\beta}{2 \varepsilon} e^{T} b\right)^{2}+\hat{v}_{\hat{y}}^{2} \geq(1-a) \hat{v}_{\hat{y} \hat{y}}^{2}+\left\{1+\left(1-\frac{1}{a}\right)\left[\frac{\beta}{2 \varepsilon} e^{T} b\right]^{2}\right\} \hat{v}_{\hat{y}}^{2},
$$

which is true for all positive constants $a$.

If we choose $a=C^{2} /\left[2+C^{2}\right]$ with $C$ from (6.2), there hold $0<a<1$ and, since (6.2),

$$
1+\left(1-\frac{1}{a}\right)\left[\frac{\beta}{2 \varepsilon} e^{T} b\right]^{2} \geq \frac{1}{2}
$$

Altogether, this implies

$$
\hat{v}_{\hat{y}}^{2}+\left(\hat{v}_{\hat{y} \hat{y}}+\hat{v}_{\hat{y}} \frac{\beta}{2 \varepsilon} e^{T} b\right)^{2} \geq C_{3}\left[\hat{v}_{\hat{y}}^{2}+\hat{v}_{\hat{y} \hat{y}}^{2}\right]
$$

with a positive constant $C_{3}$ independent of $\varepsilon$ and $\beta_{P Q}$.

From this and analogous considerations the statement follows.

\subsection{Statements for the case $\left[e_{P Q}\right]^{T} b \neq 0$}

To derive error estimates it is necessary to generalize some well-known results in the space $H^{2}\left(d b_{P Q}\right)$ for the seminorm $|\cdot|_{2, d b_{P Q}}$ and the vector space $P_{1}:=\operatorname{span}\{1, x, y\}$ like the statement

$$
\inf _{p \in P_{1}\left(d b_{P Q}\right)}\|v+p\|_{2, d b_{P Q}} \leq c\left(d b_{P Q}\right)|v|_{2, d b_{P Q}} \quad \forall v \in H^{2}\left(d b_{P Q}\right)
$$


(cf. Th. 14.1 in [6]) and the Bramble-Hilbert lemma (cf. Th. 28.1 in $[6])$ to the seminorm $|\cdot|_{\varepsilon, 2, d b_{P Q}}$ and the vector space

$$
R_{1, \varepsilon}\left(\hat{d b} b_{P Q}\right):=\operatorname{span}\left\{1, F_{3, \varepsilon}(\hat{y}), \hat{x} F_{3, \varepsilon}(\hat{y})\right\}
$$

with $F_{3, \varepsilon}$ defined by $(6.9)$.

The basis is the following lemma, which extends the well-known statement

$$
\text { For all } \hat{r} \in H^{2}\left(\hat{d}_{P Q}\right) \text { it holds: } \quad \mid \hat{r}_{2, \hat{d} b_{P Q}}=0 \quad \Longleftrightarrow \quad \hat{r} \in P_{1}\left(\hat{d}_{P Q}\right) \text {, }
$$

which is a statement for the pair $\left(|\cdot|_{2, \hat{d} b_{P Q}}, P_{1}\left(\hat{d} b_{P Q}\right)\right)$, to the pair $\left(|\cdot|_{\varepsilon, 2, \hat{d} b_{P Q}}, R_{1, \varepsilon}\left(\hat{d b} b_{P Q}\right)\right)$.

Lemma 6.3. With $R_{1, \varepsilon}\left(\hat{d} b_{P Q}\right)$ defined by (6.19) it holds for all $\hat{r} \in H^{2}\left(\hat{d}_{P Q}\right)$

$$
|\hat{r}|_{\varepsilon, 2, \hat{d} b_{P Q}}=0 \Longleftrightarrow \hat{r} \in R_{1, \varepsilon}\left(\hat{d} b_{P Q}\right) .
$$

Proof. Easy calculations show that $\hat{r} \in R_{1, \varepsilon}\left(\hat{d} b_{P Q}\right)$ yield $|\hat{r}|_{\varepsilon, 2, \hat{d} b_{P Q}}=0$.

(A) $\hat{r} \in C^{2}\left(\hat{d}_{P Q}\right)$.

Let $|\hat{r}|_{\varepsilon, 2, \hat{d} b_{P Q}}=0$ be satisfied, i.e. we have

$$
\left\|\hat{r}_{\hat{x} \hat{x}}\right\|_{0, \hat{d} b_{P Q}}=0, \quad\left\|2 \varepsilon \hat{r}_{\hat{x} \hat{y}}+\hat{r}_{\hat{x}} \beta e^{T} b\right\|_{0, \hat{d} b_{P Q}}=0 \quad \text { and } \quad\left\|2 \varepsilon \hat{r}_{\hat{y} \hat{y}}+\hat{r}_{\hat{y}} \beta e^{T} b\right\|_{0, \hat{d} b_{P Q}}=0 .
$$

The last two conditions imply that $2 \varepsilon \hat{r}_{\hat{y}}+\hat{r} \beta e^{T} b$ is constant, so that $\hat{r}$ has the form

$$
\hat{r}(\hat{x}, \hat{y})=C_{1}+C_{2}(\hat{x}) F_{3, \varepsilon}(\hat{y}) .
$$

Hence, $\left\|\hat{r}_{\hat{x} \hat{x}}\right\|_{0, \hat{d} b_{P Q}}=0$ results in $C_{2}(\hat{x})=C_{3}+C_{4} \hat{x}$ and, therefore, in $\hat{r} \in R_{1, \varepsilon}\left(\hat{d} b_{P Q}\right)$.

(B) $\hat{r} \in H^{2}\left(\hat{d b}_{P Q}\right)$.

We define the variable transformation $\breve{Z}=(\breve{x}, \breve{y})^{T}=\breve{F}(\hat{Z})$ with $\hat{Z}=(\hat{x}, \hat{y})^{T}$ by

$$
\breve{x}:=\hat{x}, \breve{y}:=\exp \left(\hat{y} \frac{\beta}{2 \varepsilon} e^{T} b\right) \quad \Longleftrightarrow \hat{x}=\breve{x}, \hat{y}=\frac{2 \varepsilon}{\beta} \frac{1}{e^{T} b} \ln (\breve{y}),
$$

which maps $\hat{d b}_{P Q}$ into $\breve{d b} b_{P Q}:=\breve{F}\left(\hat{d b}_{P Q}\right)$.

Now, corresponding to $\hat{r}$ we define the function $\breve{r}$ by

$$
\breve{r}(\breve{x}, \breve{y}):=\hat{r}(\hat{x}, \hat{y}) \exp \left(\hat{y} \frac{\beta}{2 \varepsilon} e^{T} b\right) \quad \Longleftrightarrow \quad \hat{r}(\hat{x}, \hat{y})=\breve{r}(\breve{x}, \breve{y}) \frac{1}{\breve{y}} .
$$

Easy calculations prove that

$$
\breve{r}_{\breve{x} \breve{x}}=\hat{r}_{\hat{x} \hat{x}} \exp \left(\hat{y} \frac{\beta}{2 \varepsilon} e^{T} b\right), \quad \breve{r}_{\breve{x} \breve{y}}=\frac{1}{\beta e^{T} b}\left\{2 \varepsilon \hat{r}_{\hat{x} \hat{y}}+\hat{r}_{\hat{x}} \beta e^{T} b\right\}
$$

and

$$
\breve{r}_{\breve{y} \breve{y}}=\frac{2 \varepsilon}{\left[\beta e^{T} b\right]^{2}}\left\{2 \varepsilon \hat{r}_{\hat{y} \hat{y}}+\hat{r}_{\hat{y}} \beta e^{T} b\right\} \exp \left(-\hat{y} \frac{\beta}{2 \varepsilon} e^{T} b\right),
$$


from which we get

$$
\hat{r} \in H^{2}\left(\hat{d} b_{P Q}\right) \Longleftrightarrow \breve{r} \in H^{2}\left(\breve{d} b_{P Q}\right) \quad \text { and } \quad|\hat{r}|_{\varepsilon, 2, \hat{d} b_{P Q}}=0 \Longleftrightarrow|\breve{r}|_{2, \breve{d b} b_{P Q}}=0 \text {. }
$$

The above transformation and (6.20) prove the lemma.

Remark 6.4. Part (B) proves Lemma 6.3. Part (A) only motivates the choice of $R_{1, \varepsilon}\left(\hat{d}_{P Q}\right)$.

Lemma 6.4. Assuming (6.2), then with $R_{1, \varepsilon}\left(\hat{d}_{P Q}\right)$ defined by (6.19) there exists a constant $c=c\left(\hat{d} b_{P Q}\right)$ independent of $\varepsilon$ and $\beta_{P Q}$, such that

$$
\inf _{\hat{r} \in R_{1, \varepsilon}\left(\hat{d} b_{P Q}\right)}\|\hat{v}+\hat{r}\|_{\varepsilon, 2, \hat{d} b_{P Q}} \leq c|\hat{v}|_{\varepsilon, 2, \hat{d} b_{P Q}} \quad \forall \hat{v} \in H^{2}\left(\hat{d} b_{P Q}\right) .
$$

Proof. Using Lemmas 6.2 and 6.3, for a fixed $\varepsilon$ the proof follows that of Theorem 14.1 in [6], where $P_{1}\left(\hat{d}_{P Q}\right)$ has to be replaced by $R_{1, \varepsilon}\left(\hat{d} b_{P Q}\right)$ defined by (6.19). Because of assumption (6.2) the constant $c$ is independent of $\varepsilon$ and $\beta_{P Q}$.

Lemma 6.5. Let $\hat{l}_{\varepsilon}$ be a linear form on $H^{2}\left(\hat{d} b_{P Q}\right)$, which is continuous with respect to the norm $\|\cdot\|_{\varepsilon, 2, \hat{d} b_{P Q}}$ and which satisfies

$$
\hat{l}_{\varepsilon}(\hat{r})=0 \quad \forall \hat{r} \in R_{1, \varepsilon}\left(\hat{d b}_{P Q}\right)
$$

where $R_{1, \varepsilon}\left(\hat{d} b_{P Q}\right)$ is defined by (6.19).

Assuming (6.2), then there exists a constant $c=c\left(\hat{d} b_{P Q}\right)$ independent of $\varepsilon$ and $\beta_{P Q}$, such that

$$
\left|\hat{l}_{\varepsilon}(\hat{v})\right| \leq c\left\|\hat{l}_{\varepsilon}\right\|_{\varepsilon, 2, \hat{d} b_{P Q}}^{\prime}|\hat{v}|_{\varepsilon, 2, \hat{d} b_{P Q}} \quad \forall \hat{v} \in H^{2}\left(\hat{d} b_{P Q}\right)
$$

where $\|\cdot\|_{\varepsilon, 2, \hat{d} b_{P Q}}^{\prime}$ is the norm in the dual space of $H^{2}\left(\hat{d} b_{P Q}\right)$ normed by $\|\cdot\|_{\varepsilon, 2, \hat{d} b_{P Q}}$.

Proof. The proof is substantially the same as for the standard second Bramble-Hilbert lemma. Therefore, we refer to [24], where the details are given.

We conclude this part with the following result. The change of variables $Z=G \hat{Z}+g$ and the related correspondence between functions $q, \hat{q}$ given in Section 6.2 result in

$$
\begin{gathered}
\mathrm{d} \Omega=\operatorname{det}(G) \mathrm{d} \hat{\Omega}, \\
G^{-1} e_{P Q}=\frac{2}{\beta_{P Q}} E_{\hat{y}}, \quad G^{-1} t_{P Q}=\frac{2}{\beta_{P Q}} E_{\hat{x}}, \quad \operatorname{grad} q=\left[G^{-1}\right]^{T} \operatorname{grad} \hat{q} \quad \text { and } \quad H_{q}=\left[G^{-1}\right]^{T} H_{\hat{q}} G^{-1}
\end{gathered}
$$

(where $E_{\hat{x}}=(1,0)^{T}$ and $E_{\hat{y}}=(0,1)^{T}$ denote the unit vectors in the $\hat{x}$ - and $\hat{y}$-direction), from which it follows that

$$
\left[e_{P Q}\right]^{T} \operatorname{grad} q=\frac{2}{\beta_{P Q}} \hat{q}_{\hat{y}} \quad \text { and } \quad\left[e_{P Q}\right]^{T} H_{q} e_{P Q}=\frac{4}{\left[\beta_{P Q}\right]^{2}} \hat{q}_{\hat{y} \hat{y}}
$$




\section{Estimations}

\subsection{Estimation of the approximation error}

In this section we deal with the estimation of the approximation error terms

$$
\left\|u-P_{h}^{1}(u)\right\|_{h 1} \quad \text { and } \quad\left\|u-P_{h}^{2}(u)\right\|_{h 2}
$$

with a function $u \in H^{2}(\Omega) \cap H_{0}^{1}(\Omega)$ and, at first, we consider $\left\|u-P_{h}^{1}(u)\right\|_{h 1}$.

In the following $\Pi=\Pi_{d b_{P Q}}$ denotes the restriction of $P_{h}^{1}$ to $d b_{P Q}$, i.e. there holds $\Pi: H^{2}\left(d b_{P Q}\right) \rightarrow$ $L K_{\varepsilon}(P, Q)$ with $L K_{\varepsilon}(P, Q)$ defined by (2.5). Hence, we have

$$
\left\|u-P_{h}^{1}(u)\right\|_{h 1}=\left(\frac{1}{\varepsilon} \sum_{d b_{P Q} \in d B_{h}} \iint_{d b_{P Q}}\left\{\left[e_{P Q}\right]^{T}\left[\varepsilon \operatorname{grad}\left(u-\Pi_{d b_{P Q}} u\right)+b\left(u-\Pi_{d b_{P Q}} u\right)\right]\right\}^{2} \mathrm{~d} \Omega\right)^{\frac{1}{2}}
$$

With the seminorm in the Hilbert space $\tilde{H}^{1}\left(d b_{P Q}\right)$ defined by $(6.13)$ we can also write

$$
\left\|u-P_{h}^{1}(u)\right\|_{h 1}=\left\{\varepsilon \sum_{d b_{P Q} \in d B_{h}}\left|u-\Pi_{d b_{P Q}} u\right|_{\varepsilon, \tilde{H}^{1}\left(d b_{P Q}\right)}^{2}\right\}^{\frac{1}{2}} .
$$

Since we want to follow standard techniques for error estimations we introduce the mapping $\hat{\Pi}: H^{2}\left(\hat{d} b_{P Q}\right) \rightarrow$ $L \hat{K}_{\varepsilon}(P, Q)$ by $\hat{\Pi} \hat{v}=(\Pi v)^{\wedge}$ with $v$ and $\hat{v}$ from $(6.7)$, whereas $L \hat{K}_{\varepsilon}(P, Q)$ is defined by $(6.8)$.

Lemma 7.1. Let $\hat{d}_{P Q}$ and $d b_{P Q}$ be two affine-equivalent domains.

Then it holds

$$
|v|_{\varepsilon, \tilde{H}^{1}\left(d b_{P Q}\right)}=|\hat{v}|_{\varepsilon, \tilde{H}^{1}\left(\hat{d} b_{P Q}\right)} \quad \forall \hat{v} \in \tilde{H}^{1}\left(\hat{d} b_{P Q}\right) .
$$

Proof. Using the change of variables $Z=G \hat{Z}+g,(6.21),(6.22)$ and (6.6a), we get

$$
|q|_{\varepsilon, \tilde{H}^{1}\left(d b_{P Q}\right)}^{2}=\operatorname{det}(G) \frac{4}{[\varepsilon \beta]^{2}} \iint_{\hat{d} b_{P Q}}\left(\hat{q}_{\hat{y}}+\hat{q} \frac{\beta}{2 \varepsilon} e^{T} b\right)^{2} \mathrm{~d} \hat{\Omega}=|\hat{q}|_{\varepsilon, \tilde{H}^{1}\left(\hat{d} b_{P Q}\right)}^{2}
$$

which is the statement.

Lemma 7.2. Assuming (6.2), then there exists a constant $c=c\left(\hat{d} b_{P Q}\right)$ independent of $\varepsilon$ and $\beta_{P Q}$, such that

$$
|\hat{v}-\hat{\Pi} \hat{v}|_{\varepsilon, \tilde{H}^{1}\left(\hat{d} b_{P Q}\right)} \leq c|\hat{v}|_{\varepsilon, 2, \hat{d} b_{P Q}} \quad \forall \hat{v} \in H^{2}\left(\hat{d} b_{P Q}\right) .
$$

Proof. The proof follows that of Theorem 15.3 in [6].

First step:

$$
|\hat{r}-\hat{\Pi} \hat{r}|_{\varepsilon, \tilde{H}^{1}\left(\hat{d}_{P Q}\right)}=0 \quad \forall \hat{r} \in R_{1, \varepsilon}\left(\hat{d} b_{P Q}\right)
$$

Easy calculations prove the statement of the first step. 
Second step:

$$
\begin{aligned}
& |\hat{I} \hat{v}|_{\varepsilon, \tilde{H}^{1}\left(\hat{d} b_{P Q}\right)} \leq c_{2}\left(\hat{d} b_{P Q}\right)\|\hat{v}\|_{\varepsilon, 2, \hat{d} b_{P Q}} \quad \forall \hat{v} \in H^{2}\left(\hat{d} b_{P Q}\right) \quad \text { and } \\
& |\hat{\Pi} \hat{v}|_{\varepsilon, \tilde{H}^{1}\left(\hat{d}_{P Q}\right)} \leq c_{2}\left(\hat{d} b_{P Q}\right)\|\hat{v}\|_{\varepsilon, 2, \hat{d} b_{P Q}} \quad \forall \hat{v} \in H^{2}\left(\hat{d} b_{P Q}\right)
\end{aligned}
$$

At first, because of Lemma 6.2 and the imbedding $H^{2}\left(\hat{d} b_{P Q}\right) \hookrightarrow C^{0}\left(\hat{d} b_{P Q}\right)$ there holds $\hat{v} \in C^{0}\left(\hat{d} b_{P Q}\right)$ for all $\hat{v} \in H^{2}\left(\hat{d}_{P Q}\right)$.

Further, by easy calculations it can be deduced that

$$
\left|\hat{\tilde{\Phi}}_{P Q}\right|_{\varepsilon, \tilde{H}^{1}\left(\hat{d}_{P Q}\right)}=\left|\hat{\tilde{\Phi}}_{Q P}\right|_{\varepsilon, \tilde{H}^{1}\left(\hat{d}_{P Q}\right)}=\frac{1}{2} B\left(\frac{\beta}{\varepsilon} e^{T} b\right) \sqrt{2 \frac{\gamma}{\beta}} .
$$

Now, using $\hat{\Pi} \hat{v}=\hat{v}(\hat{P}) \hat{\tilde{\Phi}}_{P Q}+\hat{v}(\hat{Q}) \hat{\tilde{\Phi}}_{Q P}$ and the continuity of $\hat{v}$ we obtain

$$
|\hat{\Pi} \hat{v}|_{\varepsilon, \tilde{H}^{1}\left(\hat{d}_{P Q}\right)} \leq B\left(\frac{\beta}{\varepsilon} e^{T} b\right) \sqrt{2 \frac{\gamma}{\beta}}\|\hat{v}\|_{\infty, \hat{d} b_{P Q}} .
$$

This, together with (6.2), Lemma 6.2 and the imbedding used above, results in

$$
|\hat{\Pi} \hat{v}|_{\varepsilon, \tilde{H}^{1}\left(\hat{d}_{P Q}\right)} \leq c_{2}\|\hat{v}\|_{\varepsilon, 2, \hat{d} b_{P Q}} \quad \forall \hat{v} \in H^{2}\left(\hat{d} b_{P Q}\right)
$$

with a constant $c_{2}=c_{2}\left(\hat{d b} b_{P Q}\right)$ independent of $\varepsilon$ and $\beta_{P Q}$, which proves the statement for $\hat{\Pi}$.

For the identity mapping $\hat{I}$ the statement follows from $(6.18 \mathrm{c})$ and $(6.2)$.

Third step:

$$
\inf _{\hat{r} \in R_{1, \varepsilon}\left(\hat{d} b_{P Q}\right)}\|\hat{v}+\hat{r}\|_{\varepsilon, 2, \hat{d} b_{P Q}} \leq c_{3}\left(\hat{d} b_{P Q}\right)|\hat{v}|_{\varepsilon, 2, \hat{d} b_{P Q}} \quad \forall \hat{v} \in H^{2}\left(\hat{d} b_{P Q}\right) .
$$

This is equivalent to Lemma 6.4 .

Now, because of the invariance (7.2), we obtain the identity

$$
|\hat{v}-\hat{\Pi} \hat{v}|_{\varepsilon, \tilde{H}^{1}\left(\hat{d} b_{P Q}\right)}=|(\hat{I}-\hat{\Pi})(\hat{v}+\hat{r})|_{\varepsilon, \tilde{H}^{1}\left(\hat{d} b_{P Q}\right)} \quad \forall \hat{r} \in R_{1, \varepsilon}\left(\hat{d} b_{P Q}\right) .
$$

As for the remaining part of the proof, we refer to the proof of Theorem 15.3 in [6].

Lemma 7.3. Let $\hat{d}_{P Q}$ and $d b_{P Q}$ be two affine-equivalent domains.

Then it holds

$$
|\hat{v}|_{\varepsilon, 2, \hat{d} b_{P Q}}=\frac{\beta_{P Q}}{2}|v|_{\varepsilon, 2, d b_{P Q}} \quad \forall v \in H^{2}\left(d b_{P Q}\right) .
$$

Proof. Using the change of variables $Z=G \hat{Z}+g,(6.21),(6.22)$ and (6.6a), we get

$$
\iint_{d b_{P Q}}\left(\varepsilon e^{T} H_{q} e+\left[e^{T} b\right] e^{T} \operatorname{grad} q\right)^{2} \mathrm{~d} \Omega=\frac{4}{[\beta]^{2}} \iint_{\hat{d} b_{P Q}}\left(\varepsilon \hat{q}_{\hat{y} \hat{y}}+\hat{q}_{\hat{y}} \frac{\beta}{2} e^{T} b\right)^{2} \mathrm{~d} \hat{\Omega} .
$$

Easy calculations for the other terms prove the statement. 
Remark 7.1. Theorem 15.1 in [6] is comparable with Lemma 7.3, where the equality in Lemma 7.3 is replaced by an inequality.

Moreover, a direct application of Theorem 15.1 in [6] is impossible, because in $H^{2}\left(d b_{P Q}\right)$ the norms $\|\cdot\|_{2, d b_{P Q}}$ and $\|\cdot\|_{\varepsilon, 2, d b_{P Q}}$ are equivalent; but this is not the case for the seminorms $|\cdot|_{2, d b_{P Q}}$ and $|\cdot|_{\varepsilon, 2, d b_{P Q}}$.

Obviously, the Lemmas 7.1, 7.2 and 7.3 yield:

Theorem 7.4. Let $\hat{d}_{P Q}$ and $d b_{P Q}$ be two affine-equivalent domains through the mapping $F$ defined as in Section 6.2.

Assuming (6.2), then there exists a constant $c=c\left(\hat{d} b_{P Q}\right)$ independent of $\varepsilon$ and $\beta_{P Q}$, such that

$$
|v-\Pi v|_{\varepsilon, \tilde{H}^{1}\left(d b_{P Q}\right)} \leq c \beta_{P Q}|v|_{\varepsilon, 2, d b_{P Q}} \quad \forall v \in H^{2}\left(d b_{P Q}\right) .
$$

Thereby, $\Pi=\Pi_{d b_{P Q}}$ denotes the restriction of $P_{h}^{1}$ defined by (4.10) to $d b_{P Q}$.

Corollary 7.5. Let a sequence $\left\{M_{n}\right\}$ of sets be given which satisfy the assumptions of Section 2.1 and let $\left\{d B_{n}\right\}:=\left\{d B_{h}\left(M_{n}\right)\right\}$ be the sequence of corresponding dual box partitions.

Additionally, let (6.1) and (6.2) be satisfied.

If $u \in H^{2}(\Omega) \cap H_{0}^{1}(\Omega)$, there exist positive constants $C_{1}$ and $C_{2}$ independent of $n, \varepsilon$ and $b$, such that

$$
\left\|u-P_{h}^{1}(u)\right\|_{h 1} \leq C_{1} \sqrt{\varepsilon}\left(\sum_{d b_{P Q} \in d B_{n}}\left[\beta_{P Q}\right]^{2}|u|_{\varepsilon, 2, d b_{P Q}}^{2}\right)^{\frac{1}{2}}
$$

and

$$
\left\|u-P_{h}^{2}(u)\right\|_{h 2} \leq C_{2} \sqrt{\varepsilon} \max _{d b_{P Q} \in d B_{n}} \beta_{P Q}|u|_{2, \Omega}
$$

Thereby, $\|\cdot\|_{h 1},\|\cdot\|_{h 2},|\cdot|_{\varepsilon, 2, d b_{P Q}}, P_{h}^{1}$ and $P_{h}^{2}$ are defined by (4.12), (6.14), (4.10) and (4.9), respectively.

Proof. Theorem 7.4 and $(7.1 \mathrm{~b})$ result in

$$
\left\|u-P_{h}^{1}(u)\right\|_{h 1} \leq \sqrt{\varepsilon}\left(\sum_{d b_{P Q} \in d B_{n}}\left[c\left(\hat{d} b_{P Q}\right)\right]^{2}\left[\beta_{P Q}\right]^{2}|u|_{\varepsilon, 2, d b_{P Q}}^{2}\right)^{\frac{1}{2}}
$$

(6.1) yields $\sup _{n} \max _{d b_{P Q} \in d B_{n}} c\left(\hat{d b_{P Q}}\right)=c<\infty$ and therefore

$$
\left\|u-P_{h}^{1}(u)\right\|_{h 1} \leq c \sqrt{\varepsilon}\left(\sum_{d b_{P Q} \in d B_{n}}\left[\beta_{P Q}\right]^{2}|u|_{\varepsilon, 2, d b_{P Q}}^{2}\right)^{\frac{1}{2}}
$$

which is the first estimate.

Since $b=0$ implies $V_{h}^{1}=V_{h}^{2}, P_{h}^{1}=P_{h}^{2},|\cdot|_{2, d b_{P Q}}=|\cdot|_{\varepsilon, 2, d b_{P Q}}$ and $\|\cdot\|_{h 1}=\|\cdot\|_{h 2}$, the second estimate obviously follows from the first one.

\subsection{Estimation of the consistency error}

In this section we deal with the estimation of the consistency error term

$$
\sup _{w_{h} \in V_{h}^{2}} \frac{\left|a_{h}\left(u, w_{h}\right)-d_{h}\left(w_{h}\right)\right|}{\left\|w_{h}\right\|_{h 2}}
$$


where $u$ is the solution of (2.1) with $u \in H^{2}(\Omega) \cap H_{0}^{1}(\Omega)$.

Therefore, for $w_{h} \in V_{h}^{2}$ we study

$$
\delta_{h}\left(u, w_{h}\right):=a_{h}\left(u, w_{h}\right)-d_{h}\left(w_{h}\right) .
$$

Remark 7.2. The approach in this section is substantially the same as for the Poisson equation in [23]. Therefore, we give only the main ideas. For the reader who is interested in more details we refer to [24].

Since $u$ is a solution in a weak sense, we obtain with $(\mathrm{A})$ and $w_{h}(P)=0$ for $P \in M_{b}$

$$
-\sum_{b_{P} \in B_{h}} w_{h}(P) \iint_{b_{P}} f \mathrm{~d} \Omega=\sum_{d b_{P Q} \in d B_{h}}\left[w_{h}(P)-w_{h}(Q)\right] \int_{b_{P} \cap b_{Q}}\left[e_{P Q}\right]^{T}[\varepsilon \operatorname{grad} u+b u] \mathrm{d} \Gamma .
$$

Further, from (4.13) we infer in $\operatorname{Int}\left(d b_{P Q}\right)$ that

$$
[\varepsilon \operatorname{grad} u+b u]^{T} \operatorname{grad} w_{h}=\frac{w_{h}(Q)-w_{h}(P)}{\beta_{P Q}}\left[e_{P Q}\right]^{T}[\varepsilon \operatorname{grad} u+b u] \quad \forall w_{h} \in V_{h}^{2} .
$$

Hence, using $d b_{P Q}^{12}$ defined by (6.12) we have

$$
\delta_{h}\left(u, w_{h}\right)=\sum_{d b_{P Q} \in d B_{h}} \delta_{d b_{P Q}}\left(u, w_{h}\right):=\sum_{d b_{P Q} \in d B_{h}}\left[w_{h}(Q)-w_{h}(P)\right] \eta_{d b_{P Q}}(u)
$$

with

$$
\eta_{d b_{P Q}}(u):=\frac{1}{\beta_{P Q}} \iint_{d b_{P Q}}\left[e_{P Q}\right]^{T}[\varepsilon \operatorname{grad} u+b u] \mathrm{d} \Omega-\frac{1}{2} \int_{d b_{P Q}^{12}}\left[e_{P Q}\right]^{T}[\varepsilon \operatorname{grad} u+b u] \mathrm{d} \Gamma .
$$

Further, we define $\eta_{\hat{d}_{P Q}}$ by

$$
\eta_{\hat{d} b_{P Q}}(\hat{u}):=\frac{1}{2}\left\{\iint_{\hat{d} b_{P Q}}\left(\varepsilon \hat{u}_{\hat{y}}+\hat{u} \frac{\beta_{P Q}}{2}\left[e_{P Q}\right]^{T} b\right) \mathrm{d} \hat{\Omega}-\int_{\hat{d b}_{P Q}^{12}}\left(\varepsilon \hat{u}_{\hat{y}}+\hat{u} \frac{\beta_{P Q}}{2}\left[e_{P Q}\right]^{T} b\right) \mathrm{d} \hat{\Gamma}\right\} .
$$

Lemma 7.6. Let $\hat{d} b_{P Q}$ and $d b_{P Q}$ be two affine-equivalent domains.

Then it holds

$$
\eta_{d b_{P Q}}(v)=\eta_{\hat{d} b_{P Q}}(\hat{v}) \quad \forall \hat{v} \in H^{2}\left(\hat{d}_{P Q}\right) .
$$

Lemma 7.7. Assuming (6.2), then there exists a constant $c=c\left(\hat{d} b_{P Q}\right)$ independent of $\varepsilon$ and $\beta_{P Q}$, such that

$$
\left|\eta_{\hat{d} b_{P Q}}(\hat{v})\right| \leq c \varepsilon|\hat{v}|_{\varepsilon, 2, \hat{d} b_{P Q}} \quad \forall \hat{v} \in H^{2}\left(\hat{d}_{P Q}\right)
$$

Proof. To prove the statement, we define a linear form $\hat{l}_{\varepsilon}$ on $H^{2}\left(\hat{d} b_{P Q}\right)$ by

$$
\hat{l}_{\varepsilon}(\hat{v}):=\frac{2}{\varepsilon} \eta_{\hat{d} b_{P Q}}(\hat{v})=\iint_{\hat{d} b_{P Q}}\left(\hat{v}_{\hat{y}}+\hat{v} \frac{\beta_{P Q}}{2 \varepsilon}\left[e_{P Q}\right]^{T} b\right) \mathrm{d} \hat{\Omega}-\int_{\hat{d} b_{P Q}^{12}}\left(\hat{v}_{\hat{y}}+\hat{v} \frac{\beta_{P Q}}{2 \varepsilon}\left[e_{P Q}\right]^{T} b\right) \mathrm{d} \hat{\Gamma} .
$$


Easy calculations show for the basis functions in $R_{1, \varepsilon}\left(\hat{d} b_{P Q}\right)$ that

$$
\frac{\partial}{\partial \hat{y}}[1]+[1] \frac{\beta}{2 \varepsilon} e^{T} b=\frac{\beta}{2 \varepsilon} e^{T} b, \quad \frac{\partial}{\partial \hat{y}}\left[F_{3, \varepsilon}(\hat{y})\right]+\left[F_{3, \varepsilon}(\hat{y})\right] \frac{\beta}{2 \varepsilon} e^{T} b=0
$$

and

$$
\frac{\partial}{\partial \hat{y}}\left[\hat{x} F_{3, \varepsilon}(\hat{y})\right]+\left[\hat{x} F_{3, \varepsilon}(\hat{y})\right] \frac{\beta}{2 \varepsilon} e^{T} b=0
$$

from which it follows that $\hat{l}_{\varepsilon}(\hat{r})=0 \quad \forall \hat{r} \in R_{1, \varepsilon}\left(\hat{d}_{P Q}\right)$ with (6.11).

Therefore, using (7.5) and Lemma 6.5 we get the desired result.

Obviously, the Lemmas 7.6, 7.7 and 7.3 result in:

Theorem 7.8. Let $\hat{d}_{P Q}$ and $d b_{P Q}$ be two affine-equivalent domains through the mapping $F$ defined as in Section 6.2 .

Assuming (6.2), then there exists a constant $c=c\left(\hat{d} b_{P Q}\right)$ independent of $\varepsilon$ and $\beta_{P Q}$, such that

$$
\left|\delta_{d b_{P Q}}\left(v, w_{h}\right)\right| \leq c \varepsilon \beta_{P Q}|v|_{\varepsilon, 2, d b_{P Q}}\left|w_{h}(P)-w_{h}(Q)\right| \quad \forall v \in H^{2}\left(d b_{P Q}\right), w_{h} \in \tilde{P}(P, Q)
$$

Thereby, $\delta_{d b_{P Q}}$ is defined by (7.3).

Corollary 7.9. Let a sequence $\left\{M_{n}\right\}$ of sets be given which satisfy the assumptions of Section 2.1 and let $\left\{B_{n}\right\}:=\left\{B_{h}\left(M_{n}\right)\right\}$ and $\left\{d B_{n}\right\}:=\left\{d B_{h}\left(M_{n}\right)\right\}$ be the sequence of corresponding box and dual partitions, respectively.

Additionally, let (6.1) and (6.2) be satisfied.

If $u \in H^{2}(\Omega) \cap H_{0}^{1}(\Omega)$ is the solution of $(2.1)$, there exists a positive constant $c$ independent of $n, \varepsilon$ and $b$, such that

$$
\sup _{w_{h} \in V_{h}^{2}} \frac{\left|a_{h}\left(u, w_{h}\right)-d_{h}\left(w_{h}\right)\right|}{\left\|w_{h}\right\|_{h 2}} \leq c \sqrt{\varepsilon}\left\{\sum_{d b_{P Q} \in d B_{n}}\left[\beta_{P Q}\right]^{2}|u|_{\varepsilon, 2, d b_{P Q}}^{2}\right\}^{\frac{1}{2}}
$$

Thereby, $a_{h}, d_{h}, V_{h}^{2},\|\cdot\|_{h 2}$ and $|\cdot|_{\varepsilon, 2, d b_{P Q}}$ are defined by (2.8), (2.7), (2.3), (4.12b) and (6.14), respectively.

Proof. Using Theorem 7.8 and (6.1), for a solution $u$ of (2.1) and all $w_{h}$ in $V_{h}^{2}$ in the same way as in the proof of Corollary 7.5 we obtain

$$
\left|\delta_{h}\left(u, w_{h}\right)\right| \leq c \varepsilon \sum_{d b_{P Q} \in d B_{n}} \beta_{P Q}|u|_{\varepsilon, 2, d b_{P Q}}\left|w_{h}(P)-w_{h}(Q)\right| .
$$

The rest is easy to prove and results in the statement. Thereby,

$$
\left\{\frac{\varepsilon}{2} \sum_{d b_{P Q} \in d B_{n}}\left[w_{h}(P)-w_{h}(Q)\right]^{2}\right\}^{\frac{1}{2}} \leq \sqrt{\frac{1}{c_{1}}}\left\|w_{h}\right\|_{h 2}
$$

is used, which holds because of (6.1) and (4.15).

Acknowledgements. The author would like to thank the anonymous referees for many useful and helpful suggestions. 


\section{REFERENCES}

[1] L. Angermann, Error Estimate for the Finite-Element Solution of an Elliptic Singularly Perturbed Problem. IMA J. Numer. Anal. 15 (1995) 161-196.

[2] R.E. Bank, J.F. Bürgler, W. Fichtner and R.K. Smith, Some Upwinding Techniques for Finite Element Approximations of Convection-Diffusion Equations. Numer. Math. 58 (1990) 185-202.

[3] R.E. Bank, W.M. Jr. Coughran and L.C. Cowsar, The Finite Volume Scharfetter-Gummel Method for Steady Convection Diffusion Equations. Comput. Visual Sci. 1 (1998) 123-136.

[4] J. Baranger, J.-F. Maitre and F. Oudin, Connection between Finite Volume and Mixed Finite Element Methods. RAIRO Modél. Math. Anal. Numér. 30 (1996) 445-465.

[5] D. Braess, Finite Elemente. Springer, Berlin (1992).

[6] P.G. Ciarlet, Basic Error Estimates for Elliptic Problems, in Handbook of Numerical Analysis, Vol. II, Part 1, P.G. Ciarlet and J.L. Lions Eds., Elsevier, Amsterdam (1991) 17-351

[7] R. Eymard, T. Gallouet and R. Herbin, Convergence of Finite Volume Schemes for Semilinear Convection Diffusion Equations. Numer. Math. 1 (1999) 1-26.

[8] E. Gatti, S. Micheletti and R. Sacco, A New Galerkin Framework for the Drift-Diffusion Equation in Semiconductors. East-West J. Numer. Math. 6 (1998) 101-135.

[9] B. Heinrich, Finite Difference Methods on Irregular Networks. A Generalized Approach to Second Order Problems. Akademie, Berlin (1987).

[10] R. Herbin, An Error Estimate for a Finite Volume Scheme for a Diffusion-Convection Problem on a Triangular Mesh. Numer. Methods Partial Differential Equations 11 (1995) 165-173.

[11] R.D. Lazarov and I.D. Mishev, Finite Volume Methods for Reaction-Diffusion Problems, in Finite Volumes for Complex Applications, F. Benkhaldoun and R. Vilsmeier Eds., Hermes, Paris (1996) 231-240.

[12] J.J.H. Miller and S. Wang, A New Non-Conforming Petrov-Galerkin Finite Element Method with Triangular Elements for an Advection-Diffusion Problem. IMA J. Numer. Anal. 14 (1994) 257-276.

[13] I.D. Mishev, Finite Volume and Finite Volume Element Methods for Nonsymmetric Problems. Ph.D. thesis, Texas A\&M University (1996).

[14] K.W. Morton, Numerical Solution of Convection-Diffusion Problems. Chapman and Hall, London (1996).

[15] K.W. Morton, M. Stynes and E. Süli, Analysis of a Cell-Vertex Finite Volume Method for Convection-Diffusion Problems. Math. Comp. 66 (1997) 1369-1406.

[16] H.G. Roos, M. Stynes and L. Tobiska, Numerical Methods for Singularly Perturbed Differential Equations. Springer, London (1996).

[17] R. Sacco and M. Stynes, Finite Element Methods for Convection-Diffusion Problems Using Exponential Splines on Triangles. Comput. Math. Appl. 35 (1998) 35-45.

[18] R. Sacco, E. Gatti and L. Gotusso, A Nonconforming Exponentially Fitted Finite Element Method for Two-Dimensional Drift-Diffusion Models in Semiconductors. Numer. Methods Partial Differential Equations 15 (1999) 133-150.

[19] H.-P. Scheffler and R. Vanselow, Convergence Analysis of a Cell-Centered FVM, in Finite Volumes for Complex Applications II, R. Vilsmeier, F. Benkhaldoun and D. Hänel Eds., Hermes, Paris (1999) 181-188.

[20] L.L. Schumaker, Spline Functions: Basic Theory. Wiley, New York (1981).

[21] S. Selberherr, Analysis and Simulation of Semiconductor Devices. Springer, Wien (1984).

[22] G. Strang, Variational Crimes in the Finite Element Method, in The Mathematical Foundations of the Finite Element Method with Applications to Partial Differential Equations, A.K. Aziz Ed., Academic Press (1972) 689-710.

[23] R. Vanselow and H.-P. Scheffler, Convergence Analysis of a Finite Volume Method via a New Nonconforming Finite Element Method. Numer. Methods Partial Differential Equations 14 (1998) 213-231.

[24] R. Vanselow, Convergence Analysis for an Exponentially Fitted FVM. Preprint MATH-NM-09-99, TU Dresden (1999).

[25] J. Xu and L. Zikatanov, A Monotone Finite Element Scheme for Convection-Diffusion Equations. Math. Comp. 68 (1999) $1429-1446$.

To access this journal online:

www.edpsciences.org 